\title{
Fermi-LAT $\boldsymbol{\gamma}$-ray anisotropy and intensity explained by unresolved Radio-Loud Active Galactic Nuclei
}

\author{
Mattia Di Mauro, ${ }^{a, b, c}$ Alessandro Cuoco, ${ }^{a, b}$ Fiorenza Donato $^{a, b}$ Jennifer \\ M. Siegal-Gaskins ${ }^{d}$ \\ ${ }^{a}$ Dipartimento di Fisica, Università di Torino, via P. Giuria 1, 10125 Torino, Italy \\ ${ }^{b}$ Istituto Nazionale di Fisica Nucleare, Sezione di Torino, Via P. Giuria 1, 10125 Torino, Italy \\ ${ }^{c}$ LAPTh, Université de Savoie, CNRS, 9 Chemin de Bellevue, B.P. 110, F-74941 Annecy-le-Vieux, \\ France \\ ${ }^{d}$ California Institute of Technology, 1200 E. California Blvd., Pasadena, CA 91125, USA \\ E-mail: mattia.dimauro@to.infn.it, alessandro.cuoco@to.infn.it, \\ donato@to.infn.it, jsg@tapir.caltech.edu
}

ABSTRACT: Radio-loud active galactic nuclei (AGN) are expected to contribute substantially to both the intensity and anisotropy of the isotropic $\gamma$-ray background (IGRB). In turn, the measured properties of the IGRB can be used to constrain the characteristics of proposed contributing source classes. We consider individual subclasses of radio-loud AGN, including low-, intermediate-, and high-synchrotron-peaked BL Lacertae objects, flat-spectrum radio quasars, and misaligned AGN. Using updated models of the $\gamma$-ray luminosity functions of these populations, we evaluate the energy-dependent contribution of each source class to the intensity and anisotropy of the IGRB. We find that collectively radio-loud AGN can account for the entirety of the IGRB intensity and anisotropy as measured by the Fermi Large Area Telescope (LAT). Misaligned AGN provide the bulk of the measured intensity but a negligible contribution to the anisotropy, while high-synchrotronpeaked BL Lacertae objects provide the dominant contribution to the anisotropy. In anticipation of upcoming measurements with the Fermi-LAT and the forthcoming Cherenkov Telescope Array, we predict the anisotropy in the broader energy range that will be accessible to future observations. 


\section{Contents}

1 Introduction 1

2 Anisotropy Model Predictions $\quad 4$

2.1 The Angular Anisotropy 4

2.2 Luminosity Function and the Source Count Distribution 4

2.3 Energy range rescaling of the cumulative source count distribution 6

$2.4 d^{2} N /(d S d \Gamma)$ energy range rescaling 8

$\begin{array}{lll}3 & \text { Results } & 10\end{array}$

4 Predicted anisotropy for CTA $\quad 16$

$\begin{array}{lll}4.1 & \text { Discussion } & 21\end{array}$

5 Conclusions $\quad 22$

\section{Introduction}

The intensity and anisotropy of a diffuse $\gamma$-ray background encode information about its contributing sources. The isotropic $\gamma$-ray background (IGRB) is the diffuse residual $\gamma$-ray emission, apparent especially at high Galactic latitudes, observed when the Galactic diffuse emission is subtracted from the observed $\gamma$-ray sky, and when resolved point sources are either subtracted or masked. The origin of this emission is not yet fully understood, but it is thought to originate from unresolved sources of extragalactic and possibly Galactic origin. Recent measurements of the intensity [1] and angular power spectrum [2] of the IGRB by the Fermi Large Area Telescope (LAT) have enabled more detailed studies of the contributors to this emission.

The intensity spectrum of the IGRB is largely consistent with a single power law in the energy range of $200 \mathrm{MeV}-100 \mathrm{GeV}$ [2]. However, it is expected that many $\gamma$-ray source classes contribute to the IGRB over this energy range, including $\gamma$-ray emitting classes of active galactic nuclei (AGN) [3-10], star-forming galaxies [11-15], Galactic millisecond pulsars (MSPs) [16-18], as well as proposed source classes such as annihilating or decaying dark matter [19-23].

The Fermi-LAT, using approximately 2 years of data, has measured the angular power spectrum of the diffuse emission at Galactic latitudes $|b|>30^{\circ}$, in four energy bins spanning 1 to $50 \mathrm{GeV}$ [2]. At multipoles $l \geq 155$, an angular power above the photon noise level is detected at $>99.99 \% \mathrm{CL}$ in the $1-2 \mathrm{GeV}, 2-5 \mathrm{GeV}$, and 5-10 GeV energy bins, and at $>99 \% \mathrm{CL}$ in the 10-50 GeV energy range. Within each energy bin, the measured angular power takes approximately the same value at all multipoles, suggesting that it originates 
from the contribution of one or more unclustered point source populations. We denote this multipole-independent anisotropy as a function of energy $C_{\mathrm{P}}(E)$.

In this work we predict both the intensity of the anisotropy and its energy dependence according to the most recent $\gamma$-ray emission models of radio-loud AGN, and compare the results to the Fermi-LAT data. Radio-loud AGN sources are a small fraction of AGN (15-20\%) but are the most powerful ones, with a ratio of radio (at $5 \mathrm{GHz}$ ) to optical (Bband) flux greater than 10 [24]. This category of AGN is divided into blazars or misaligned (MAGN) sources according to the angle of the jets with respect to the line of sight (los). Blazars (MAGN) are objects with an emission angle smaller (larger) than about $14^{\circ}$ [25]. Furthermore, blazars are traditionally divided into flat spectrum radio quasars (FSRQs) and BL Lacertae (BL Lac) objects according to the presence or absence of strong broad emission lines in their optical/UV spectrum, respectively [25, 26]. Extending the classification proposed for BL Lacs [27], all blazars could also be divided according to the value of the synchrotron-peak frequency $\nu_{\mathrm{S}}$ of their spectrum. The low-synchrotron-peaked (LSP) blazars have the observed peak frequency in the far-infrared (IR) or IR band $\left(\nu_{\mathrm{S}}<10^{14}\right.$ $\mathrm{Hz}$ ), intermediate-synchrotron-peaked (ISP) blazars have $\nu_{\mathrm{S}}$ in the near-IR to ultraviolet (UV) frequencies $\left(10^{14} \mathrm{~Hz}<\nu_{\mathrm{S}}<10^{15} \mathrm{~Hz}\right)$, while for high-synchrotron-peaked (HSP) blazars the peak frequency is located in the UV band or at higher energies $\left(\nu_{\mathrm{S}}>10^{15}\right.$ $\mathrm{Hz}$ ) [28]. This classification is relevant also for $\gamma$-ray energies because the shape and the intensity of the spectral energy distribution at such high energies is connected to the position of the synchrotron peak: the smaller (larger) the $\nu_{\mathrm{S}}$, the softer (harder) the $\gamma$-ray photon index $\Gamma$, and the larger (smaller) the $\gamma$-ray flux $[29,30]$.

Radio-loud AGN are the most numerous population in the Fermi-LAT catalogs [3133]. Previous works have derived the redshift $z, \gamma$-ray luminosity $L_{\gamma}$, and photon index $\Gamma$ distributions for the detected sources together with predictions for the $\gamma$-ray flux from the unresolved component [3-6]. Some of the main results of those studies, which will be used in the present work, are summarized below:

1. In [3] the $\gamma$-ray emission from the MAGN population was predicted using a sample of sources detected in $\gamma$-rays and calibrated using radio data in order to construct the $\gamma$-ray luminosity function. These sources have a mean photon index of $2.37 \pm 0.32$ and a $\gamma$-ray luminosity which is about two orders of magnitude larger than the radio core luminosity at $5 \mathrm{GHz}$. The best-fit value of the unresolved emission from MAGN was found to be $25-30 \%$ of the IGRB for $E>100 \mathrm{MeV}$, enveloped in an uncertainty band of about a factor of ten.

2. The FSRQ population was analyzed in [4], where it was found that FSRQ objects are nearly all LSP blazars, with a broad redshift distribution spanning from 0.2 to 3 and a mean photon index of $2.44 \pm 0.18$. FSRQs are powerful sources with the high-energy peak of the spectral energy distribution (SED) in the range of $10 \mathrm{MeV}-1 \mathrm{GeV}$. The unresolved emission from this component contributes $9.3_{-1.0}^{+1.6 \%}$ to the IGRB for $E>100 \mathrm{MeV}$, with a steeply falling spectrum at energies above $\sim 5-10 \mathrm{GeV}$.

3. In $[5,6]$ the population of BL Lacs was studied in terms of the redshift, $\gamma$-ray lu- 
minosity, and photon index distributions. In particular, [5] studied the SED and $\gamma$-ray luminosity function separately for the LSP/ISP/HSP BL Lacs using also the high-energy $\gamma$-ray spectra measured by Imaging Atmospheric Cherenkov Telescopes (IACTs). LSP and ISP BL Lacs are found to be statistically the same $\gamma$-ray population with a mean photon index of $2.08 \pm 0.15$ and an exponential cut-off at $37_{-20}^{+85}$ $\mathrm{GeV}$, hence they are associated to a unique class called LISP (LSP+ISP). HSPs have a mean photon index of $1.86 \pm 0.16$ with an exponential cut-off at $910_{-450}^{+1100} \mathrm{GeV}$. The $\gamma$-ray emission from unresolved BL Lac sources was derived in $[5,6]$ to be about $7-11 \%$ of the IGRB in the range $100 \mathrm{MeV}-1 \mathrm{GeV}$, and as much as $100 \%$ for energies higher than $100 \mathrm{GeV}$.

While the anisotropy spectrum is a relatively recently available observable, historically the diffuse extragalactic $\gamma$-ray sky has been studied through the energy spectrum of the IGRB. Measurements of the spectrum of the IGRB in the energy range $200 \mathrm{MeV}-100 \mathrm{GeV}$ have been reported by the Fermi-LAT Collaboration in [1] for $b>10^{\circ}$. More recently the Fermi-LAT Collaboration has presented preliminary results for the IGRB spectrum in the broader energy range of $100 \mathrm{MeV}-820 \mathrm{GeV}$ [34]. In [5, 23] it was shown that it is possible to explain the entire spectrum of the IGRB by the unresolved emission from the FSRQ, BL Lac, MAGN, MSP, and star-forming galaxy populations.

The information available from the anisotropy measured in [2] has been used, for example, in [35] together with the source count distribution of blazars to show that they contribute only by about $20-30 \%$ to the IGRB intensity, confirming with the anisotropy the result found via the source counts alone [36]. Anisotropy from blazars has been further studied in [37, 38]. It has also been used to constrain the contribution of MSPs to the IGRB [17] showing that stronger constraints are obtained with respect to the case when intensity alone is used. A recent analysis has demonstrated that these galactic sources contribute indeed negligibly to the measured anisotropy, as well as to the IGRB intensity [16]. The anisotropy of star-forming galaxies has been studied in [39]. Finally, several works have investigated the anisotropy from dark matter annihilation into $\gamma$-rays [40-48].

In this work we compare radio-loud AGN model predictions to both the intensity and the anisotropy of the IGRB and we will show that a coherent picture can be constructed in which radio-loud AGN account for the measured values of both of these observables. This is the first time that an attempt to simultaneously explain the $\gamma$-ray flux and anisotropy data has been pursued using a single underlying global model of the unresolved emission. In $\S 2$ we describe the models for the radio-loud AGN populations and present the calculation of their intensity and anisotropy contributions to the IGRB. We discuss the results and compare them to the measured intensity and anisotropy by the Fermi-LAT in $\S 3$. In addition to comparing model predictions for the intensity and anisotropy of the IGRB in the energy range $100 \mathrm{MeV}-100 \mathrm{GeV}$, relevant for Fermi-LAT observations, we also study the energy range $100 \mathrm{GeV}-10 \mathrm{TeV}$, as will be covered by the forthcoming Cherenkov Telescope Array (CTA) observatory [49, 50]. In $\S 4$ we derive the expected angular power from unresolved radio-loud AGN in the higher energy range relevant for future CTA observations and compare it with the expected sensitivity reach of CTA. We discuss and conclude 
in $\S 5$.

\section{Anisotropy Model Predictions}

\subsection{The Angular Anisotropy}

The angular power $C_{\mathrm{P}}$ produced by the unresolved flux of an unclustered point source population is derived using the following equation $[2,35,51]$ :

$$
C_{\mathrm{P}}\left(E_{0} \leq E \leq E_{1}\right)=\int_{\Gamma_{\min }}^{\Gamma_{\max }} d \Gamma \int_{0}^{S_{\mathrm{t}}(\Gamma)} S^{2} \frac{d^{2} N}{d S d \Gamma} d S,
$$

where $S$ is the photon flux of the source integrated in the range $E_{0} \leq E \leq E_{1}$ in units of ph $\mathrm{cm}^{-2} \mathrm{~s}^{-1}$, while $S_{\mathrm{t}}(\Gamma)$ denotes the flux detection threshold as function of the photon index of the source $\Gamma$ (see below), and where $\Gamma_{\min }-\Gamma_{\max }$ is its range of variation. Finally, $d^{2} N /(d S d \Gamma)$ is the differential number of sources per unit flux $S$, unit photon index $\Gamma$ and unit solid angle.

It is well known that a strong bias is present between the flux and the photon index of sources detected by the Fermi-LAT when considering fluxes integrated in the range $100 \mathrm{MeV}-100 \mathrm{GeV}$. Sources with a photon index of 1.5 can be detected to fluxes $(100 \mathrm{MeV}$ $-100 \mathrm{GeV}$ ) a factor of about 20 fainter than those at which a source with a photon index of 3.0 can be detected [31, 32]. This means that the function $S_{\mathrm{t}}(\Gamma)$ cannot be approximated as a constant in $\Gamma$ when considering fluxes in that energy range. On the other hand, it has been shown that the bias is almost absent if the fluxes $S$ integrated above $1 \mathrm{GeV}$ are considered $[32,35]$. In this case, the function $S_{\mathrm{t}}(\Gamma)$ can be simply approximated as a constant $S_{\mathrm{t}}(\Gamma)=S_{>1}$, where $S_{>1}$ is the flux integrated above $1 \mathrm{GeV}$. Further, as we will show in $\S 2.4$, since the source spectra are described by Eqs. 2.6, 2.7, and 2.8, a relation between $S_{>1}$ and the integrated flux between $E_{0}$ and $E_{1}$ can easily be found, and thus the function $S_{\mathrm{t}}(\Gamma)$ can be calculated for any energy range considered.

Following [35] we adopt the value $S_{>1}=5 \cdot 10^{-10} \mathrm{ph} \mathrm{cm}^{-2} \mathrm{~s}^{-1}$, which is appropriate when the 1FGL catalogue is used as reference for the resolved point sources. This is a consistent choice with respect to the Fermi-LAT anisotropy measurements, which were made after masking the 1FGL sources, and with which we compare the predicted model anisotropy.

\subsection{Luminosity Function and the Source Count Distribution}

To derive the $d^{2} N /(d S d \Gamma)$ required to calculate $C_{\mathrm{P}}$ for the MAGN, FSRQ and BL Lac sources, the quantity we will use for each population is the $\gamma$-ray luminosity function (LF) $\rho_{\gamma}\left(L_{\gamma}, z, \Gamma\right)=d N / d \Gamma d z d L_{\gamma}$ which specifies the comoving number density of the given objects, differentially per rest-frame luminosity $L_{\gamma}$, redshift $z$, and photon index $\Gamma$. The LF completely characterizes the specified source population. We will use the LFs of MAGN, FSRQ and BL Lac populations as derived in [3-5]. In these models, the LF is assumed to be separable in the $\Gamma$ variable, whose distribution is parameterized as a Gaussian function:

$$
\frac{d N}{d \Gamma} \propto \exp \left(-\frac{(\Gamma-\bar{\Gamma})^{2}}{2 \sigma^{2}}\right)
$$


with the values for the mean spectral index $\bar{\Gamma}$ and the dispersion $\sigma$ fixed, for each population, to the numbers reported in $\S 1$. With a slight abuse of notation we will also write $\rho_{\gamma}\left(L_{\gamma}, z, \Gamma\right)=d N / d \Gamma \rho_{\gamma}\left(L_{\gamma}, z\right)$.

To simplify the discussion, and also to facilitate the comparison with available data, in this section we consider the cumulative source count distribution $N(>S)$, which represents the number of sources with a flux larger than $S$. The same methods can be applied to $d^{2} N /(d S d \Gamma)$, as we briefly discuss in $\S 2.4$. The $N(>S)$ can be obtained from the LF as $[3-6]$ :

$$
N(>S)=\Delta \Omega \int_{\Gamma_{\min }}^{\Gamma_{\max }} d \Gamma \int_{z_{\min }}^{z_{\max }} d z \int_{L_{\gamma}(S, \Gamma, z)}^{L_{\gamma, \max }} d L_{\gamma} \frac{d V}{d z} \frac{d N}{d \Gamma} \rho_{\gamma}\left(L_{\gamma}, z\right)
$$

where $d V / d z$ is the comoving volume per unit redshift [52] and $\Delta \Omega$ is the solid angle. We will use in the following a $\Delta \Omega$ of $2 \pi$ corresponding to $\gamma$-ray sources above a Galactic cut of $\pm 30^{\circ}$. This is opposed to another common convention where $N(>S)$ is divided by $\Delta \Omega$ and expressed in units of $\mathrm{deg}^{-2}$. The limits of integration $\Gamma_{\min }, \Gamma_{\max }, z_{\min }, z_{\max }$, and $L_{\gamma, \max }$ are taken from [3-5], although we note that the results depend only weakly on the specific values of the limits. Finally $L_{\gamma}(S, \Gamma, z)$ represents the rest-frame $\gamma$-ray luminosity for a source with a photon index $\Gamma$ at redshift $z$ with observed photon flux $S$, and will be derived in the next section. Both $S$ and $L_{\gamma}$ refer to integrated quantities in the relevant energy range (see next section).

In Fig. 1 the theoretical source count distribution $N(>S)$ in terms of $S_{0.1-100 \mathrm{GeV}}$ is shown, together with the $1 \sigma$ uncertainty band, for MAGN [3], FSRQs [4], and BL Lac LISP (LSP+ISP) and HSP objects [5]. The $1 \sigma$ band has been derived for each AGN class considering the uncertainty on the $\gamma$-ray emission mechanism and on the redshift, $\gamma$-ray luminosity, and photon index distributions. In the case of MAGN [3], the number of detected sources in the Fermi-LAT catalogs is too small to determine a $\gamma$-ray LF, and a correlation between the $\gamma$-ray and radio emission from the core of the MAGN was performed. The $\gamma$-ray luminosity function was then derived from the radio luminosity function of [53]. The calibration of this correlation is the main source of uncertainty for the theoretical prediction of the MAGN source count distribution shown in Fig. 1. This uncertainty also leads to a large uncertainty in the prediction of the the unresolved $\gamma$-ray emission from MAGN [3]. Blazars, FSRQ, and BL Lacs are numerous in the Fermi-LAT catalogs [31-33], hence in $[4,5]$ the $\rho_{\gamma}$ was derived directly from $\gamma$-ray data. In this case the uncertainties on the source count distributions and on the unresolved $\gamma$-ray emission come from the uncertainty of the redshift, $\gamma$-ray luminosity, and photon index distributions, and of the SEDs of these sources.

In Fig. 1 we also show the experimental determinations of $N(>S)$ as derived from resolved $\gamma$-ray sources, again, normalized to the number of sources above $\pm 30^{\circ}$ in Galactic latitude, coherently with the theoretical model predictions. The data points have been taken from [3-5], and have been derived as:

$$
N_{\text {exp }}(>S)=\sum_{i=1}^{N_{S}} \frac{1}{\omega\left(S_{i}\right)},
$$



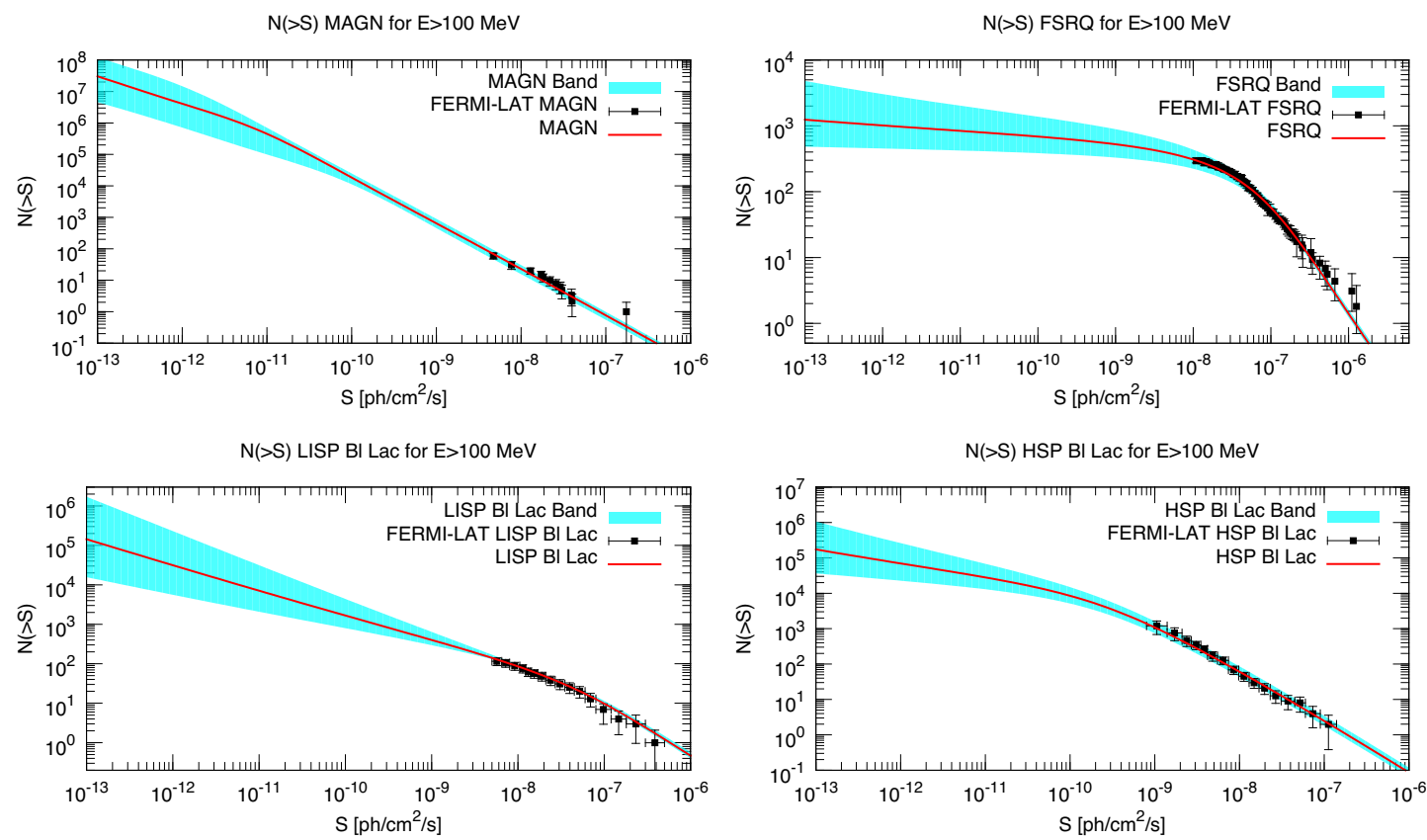

Figure 1. The theoretical cumulative source count distribution $N(>S)$ (solid red line) together with the $1 \sigma$ uncertainty band (cyan) is shown for radio-loud AGN classes as derived in [3-5]: MAGN (top left), FSRQs (top right), LISP (bottom left) and HSP (bottom right) BL Lacs. The data (black points) of the Fermi-LAT experimental source count distribution for the sources of each class are also shown, taken from [3-5].

where the sum is over the $N_{S}$ sources with a flux $S_{i}>S$, and $\omega(S)$ is the Fermi-LAT detection efficiency for a source with flux $S$ in the energy range 0.1-100 GeV. For BL Lacs and MAGN the efficiency $\omega(S)$ derived in [3] is adopted, while for FSRQs we refer to the one calculated in [36]. Uncertainties are simply given by the Poissonian errors $(\propto \sqrt{(} N))$ associated with the finite number of sources in each flux bin and by the uncertainty on the efficiency itself as given in $[3,4,6]$. The experimental source count distribution of FSRQs is based on the sources of the 1FGL catalog, while that for MAGN is based on both the 1FGL and 2FGL catalogs, and that for BL Lacs on the 2FGL catalog.

\subsection{Energy range rescaling of the cumulative source count distribution}

The source count distributions derived in [3-5] and displayed in Fig. 1 are valid in the energy range $100 \mathrm{MeV}-100 \mathrm{GeV}$, while we will need to calculate $C_{\mathrm{P}}(E)$ and therefore $d^{2} N / d S d \Gamma$ in the energy bins $[1,10],[1.04,1.99],[1.99,5.00],[5.0,10.4]$ and $[10.4,50.0] \mathrm{GeV}$. Below, we illustrate how we rescale $d^{2} N / d S d \Gamma$ to the new energy bands, again demonstrating the procedure on $N(>S)$ rather than $d^{2} N / d S d \Gamma$ itself.

The source energy spectrum $d N / d E$ is defined by:

$$
\frac{d N}{d E}\left(E, \Gamma, E_{\mathrm{c}}\right)=K \mathcal{F}\left(E, \Gamma, E_{\mathrm{c}}\right)
$$


where $K$ is a normalization factor and $\mathcal{F}\left(E, \Gamma, E_{\mathrm{c}}\right)$ is the energy-dependent part of the spectrum which depends also on the photon index $\Gamma$ and energy cutoff $E_{\mathrm{c}} . \mathcal{F}\left(E, \Gamma, E_{\mathrm{c}}\right)$ is given by a simple power law (with $E_{\mathrm{c}} \rightarrow \infty$ ) for MAGN [3], a power law with an exponential cut-off for BL Lacs as in [5], and a power law with a square-root exponential cut-off for FSRQs as in [4]:

$$
\begin{aligned}
\mathcal{F}_{\mathrm{MAGN}}(E, \Gamma) & =\left(\frac{E}{E_{\mathrm{P}}}\right)^{-\Gamma} \\
\mathcal{F}_{\mathrm{BLLAC}}\left(E, \Gamma^{\prime}, E_{\mathrm{c}}\right) & =\left(\frac{E}{E_{\mathrm{P}}^{\prime}}\right)^{-\Gamma^{\prime}} \exp \left(-\frac{E}{E_{\mathrm{c}}}\right) \\
\mathcal{F}_{\mathrm{FSRQ}}\left(E, \Gamma^{\prime \prime}, E_{\mathrm{c}}^{\prime}\right) & =\left(\frac{E}{E_{\mathrm{P}}^{\prime \prime}}\right)^{-\Gamma^{\prime \prime}} \exp \left(-\sqrt{\frac{E}{E_{\mathrm{c}}^{\prime}}}\right),
\end{aligned}
$$

where $E_{\mathrm{c}}$ and $E_{\mathrm{c}}^{\prime}$ are the cut-off energies, $E_{\mathrm{P}}, E_{\mathrm{P}}^{\prime}$ and $E_{\mathrm{P}}^{\prime \prime}$ are the pivot energies fixed to 1 $\mathrm{GeV}$ and $\Gamma, \Gamma^{\prime}$ and $\Gamma^{\prime \prime}$ are the photon indexes. From the full SED given above, the flux $S$ and the $\gamma$-ray luminosity $L_{\gamma}$ in the benchmark energy range $E \in\left[E_{0}^{\mathrm{b}}=0.1, E_{1}^{\mathrm{b}}=100\right] \mathrm{GeV}$ can be calculated as [31, 32]:

$$
\begin{aligned}
& S \equiv S\left(E_{0}^{\mathrm{b}} \leq E \leq E_{1}^{\mathrm{b}}\right)=\int_{E_{0}^{\mathrm{b}}}^{E_{1}^{\mathrm{b}}} \frac{d N}{d E} d E \\
& L_{\gamma} \equiv L_{\gamma}\left(E_{0}^{\mathrm{b}} \leq E \leq E_{1}^{\mathrm{b}}\right)=4 \pi d_{L}^{2}(z) \int_{E_{0}^{\mathrm{b}}}^{E_{1}^{\mathrm{b}}} \frac{1}{\mathcal{K}(z, \Gamma, E)} \frac{d N}{d E} E d E=L_{\gamma}(S, \Gamma, z),
\end{aligned}
$$

where $d_{L}(z)$ is the luminosity distance and $\mathcal{K}(z, \Gamma, E)$ is the $\mathrm{K}$-correction, i.e., the ratio between the observed and the rest-frame luminosity in the given energy range. For the three SEDs the K-correction can be calculated, respectively, as

$$
\begin{aligned}
\mathcal{K}(z, \Gamma) & =(1+z)^{2-\Gamma} \\
\mathcal{K}\left(z, \Gamma, E, E_{\mathrm{c}}\right) & =(1+z)^{2-\Gamma} \exp \left(-\frac{E z}{E_{\mathrm{c}}}\right) \\
\mathcal{K}\left(z, \Gamma, E, E_{\mathrm{c}}\right) & =(1+z)^{2-\Gamma} \exp \left(-\frac{(\sqrt{E(1+z)}-\sqrt{E})}{\sqrt{E_{\mathrm{c}}}}\right) .
\end{aligned}
$$

Using Eqs. 2.6-2.8 and the definitions of $S$ and $L_{\gamma}$ in Eqs. 2.9 and 2.10, we have rescaled the fluxes $S$ and luminosities $L_{\gamma}$ valid for $100 \mathrm{MeV}$ to $100 \mathrm{GeV}$ energy range into the fluxes $S^{\prime}$ and luminosities $L_{\gamma}^{\prime}$ integrated in the energy ranges of $C_{\mathrm{P}}(E)$. Formally, these relations can be written as:

$$
\begin{aligned}
& S^{\prime}\left(E_{0} \leq E \leq E_{1}\right)=\left(\frac{S}{\int_{E_{0}^{\mathrm{b}}}^{E_{1}^{\mathrm{b}}} \mathcal{F}\left(E, \Gamma, E_{\mathrm{c}}\right) d E}\right) \int_{E_{0}}^{E_{1}} \mathcal{F}\left(E, \Gamma, E_{\mathrm{c}}\right) d E=S^{\prime}\left(S, \Gamma, E_{\mathrm{c}}\right) \\
& L_{\gamma}^{\prime}\left(E_{0} \leq E \leq E_{1}\right)=\left(\frac{L_{\gamma}}{\int_{E_{0}^{\mathrm{b}}}^{E_{1}^{\mathrm{b}}} \frac{1}{\mathcal{K}(z, \Gamma, E)} E \mathcal{F}\left(E, \Gamma, E_{\mathrm{c}}\right) d E}\right) \int_{E_{0}}^{E_{1}} \frac{E \mathcal{F}\left(E, \Gamma, E_{\mathrm{c}}\right)}{\mathcal{K}(z, \Gamma, E)} d E
\end{aligned}
$$


Given the above definitions (Eqs. 2.14, 2.15), the source count distribution $N^{\prime}\left(>S^{\prime}\right)$ for the energy range $E \in\left[E_{0}, E_{1}\right]$ can be expressed as:

$$
N^{\prime}\left(>S^{\prime}\right)=\Delta \Omega \int_{\Gamma_{\min }}^{\Gamma_{\max }} d \Gamma \int_{z_{\min }}^{z_{\max }} d z \int_{L_{\gamma}\left(S\left(S^{\prime}, \Gamma\right), \Gamma, z\right)}^{L_{\gamma, \max }} d L_{\gamma} \frac{d V}{d z} \frac{d N}{d \Gamma} \rho_{\gamma}\left(L_{\gamma}, z\right)
$$

where the relation $S\left(S^{\prime}, \Gamma\right)$ can be derived from the definition of Eq. 2.14 and depends on the type of energy spectrum used. The relation $L_{\gamma}(S, \Gamma, z)$ is also spectrum dependent and is given by Eq. 2.10 .

The resulting theoretical source count distributions $N^{\prime}\left(>S^{\prime}\right)$ for the four $C_{\mathrm{P}}(E)$ energy bins used in the Fermi-LAT anisotropy measurement are shown in Figs. 2 and 3 for HSP BL Lacs and MAGN, along with the $1 \sigma$ uncertainty band. We show also the experimental data points on $N^{\prime}\left(>S^{\prime}\right)$, calculated with Eq. 2.4 and using the relation Eq. 2.14 between benchmark fluxes $\mathrm{S}$ and rescaled fluxes $\mathrm{S}$ ':

$$
N_{e x p}^{\prime}\left(>S^{\prime}\right)=\sum_{i=1}^{N_{S^{\prime}}^{\prime}} \frac{1}{\omega\left(S_{i}\left(S_{i}^{\prime}\right)\right)}
$$

The above procedure is only approximate, since in principle a new efficiency $\omega^{\prime}\left(S^{\prime}\right)$ should be evaluated for the new energy band. Alternatively, a proper conversion of $\omega$ between energy bands could be determined, however this would require starting from the full efficiency function $\omega(S, \Gamma)$ which is not available. Nonetheless, it can be seen that the agreement between the data points and the theoretical predictions is reasonable. The agreement can be seen as a cross-check of the correctness of the global rescaling procedure for the $N(>S)$. Note that for MAGNs the flux binning has been re-adjusted for each energy bin due to the scarcity of sources available. Note, further, that the source count distribution data points are used for illustrative purposes only in these figures, and are not used in any of the following calculations.

\section{$2.4 d^{2} N /(d S d \Gamma)$ energy range rescaling}

In this section we describe how we derive the rescaled double differential distribution $d^{2} N /(d S d \Gamma)$, which is the relevant quantity entering the calculation of the anisotropy term $C_{\mathrm{P}}(E)$. The quantity $d^{2} N /(d S d \Gamma)$ can be expressed in terms of the $\gamma$-ray $\operatorname{LF} \rho_{\gamma}$ as:

$$
\frac{d^{2} N}{d \Gamma d S}(S, \Gamma) \approx \frac{\Delta \Omega}{\Delta S} \int_{z_{\min }}^{z_{\max }} d z \int_{L_{\gamma}(S, \Gamma, z)}^{L_{\gamma}(S+\Delta S, \Gamma, z)} d L_{\gamma} \frac{d V}{d z} \frac{d N}{d \Gamma} \rho_{\gamma}\left(L_{\gamma}, z\right)
$$

with $\Delta S$ sufficiently small. Then, similarly to Eq. 2.16 , the rescaled $d^{2} N /\left(d \Gamma d S^{\prime}\right)$ can be expressed as:

$$
\frac{d^{2} N}{d \Gamma d S^{\prime}}\left(S^{\prime}, \Gamma\right) \approx \frac{\Delta \Omega}{\Delta S^{\prime}} \int_{z_{\min }}^{z_{\max }} d z \int_{L_{\gamma}\left(S\left(S^{\prime}, \Gamma\right), \Gamma, z\right)}^{L_{\gamma}\left(S\left(S^{\prime}+\Delta S^{\prime}, \Gamma\right), \Gamma, z\right)} d L_{\gamma} \frac{d V}{d z} \frac{d N}{d \Gamma} \rho_{\gamma}\left(L_{\gamma}, z\right),
$$

again, with $\Delta S^{\prime}$ sufficiently small. We evaluate these expressions numerically, producing a table of $d N /\left(d S^{\prime} d \Gamma\right)$ on a grid of $S^{\prime}$ and $\Gamma$ values. We also verified that choosing $\Delta S^{\prime}$ and $\Delta S$ sufficiently small, the result becomes independent of the actual chosen values. Eq. 2.1 can then be used to calculate the anisotropy in each energy band. 

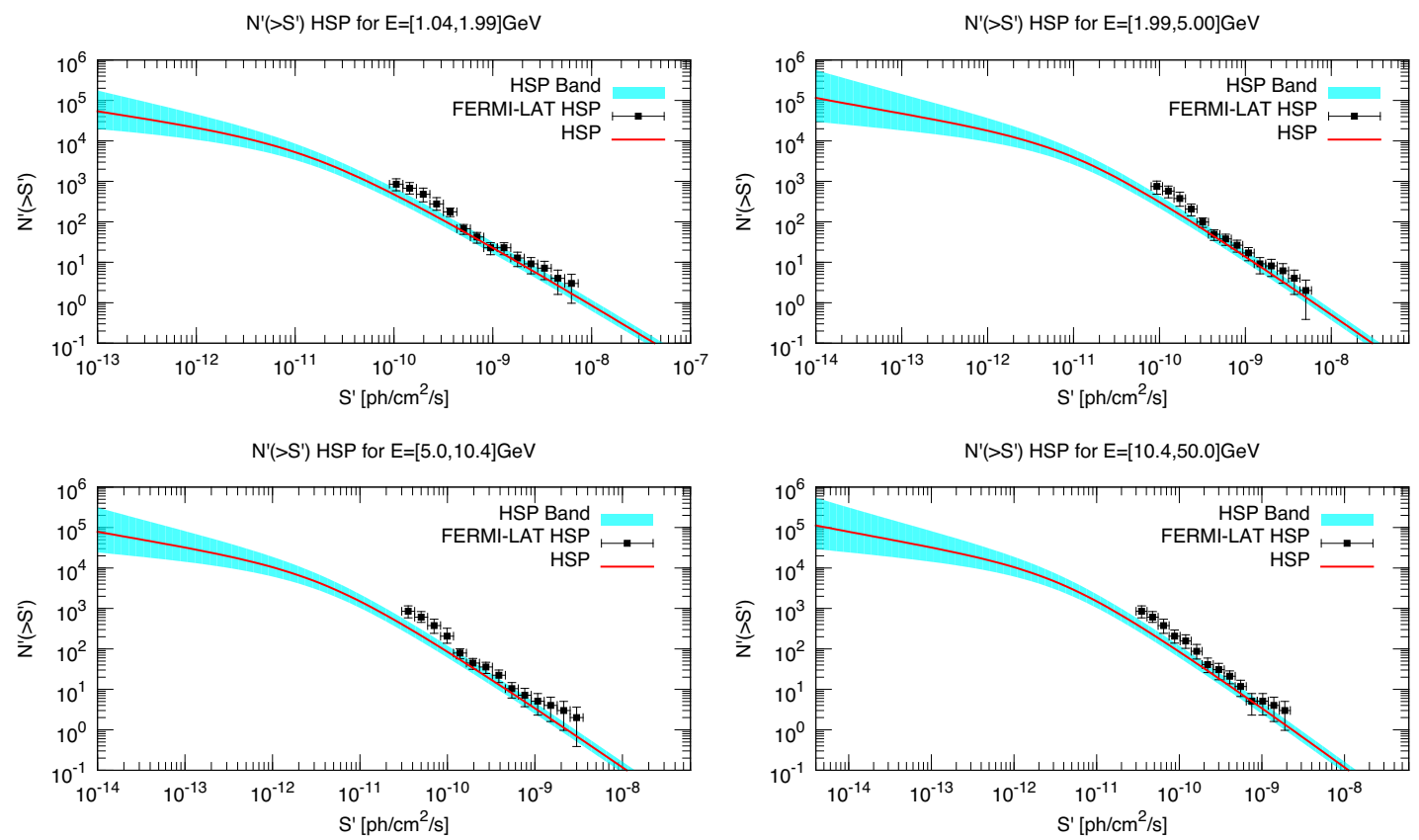

Figure 2. The HSP BL Lac theoretical cumulative source count distribution (solid red line) and the $1 \sigma$ uncertainty band (cyan) are shown for the energy bands [1.04,1.99], [1.99,5.00], [5.0,10.4] and $[10.4,50.0] \mathrm{GeV}$ (clockwise from top left). We overlay also the Fermi-LAT experimental data (black points) as derived in [3-5] and adapted to the displayed energy bins as described in the text.
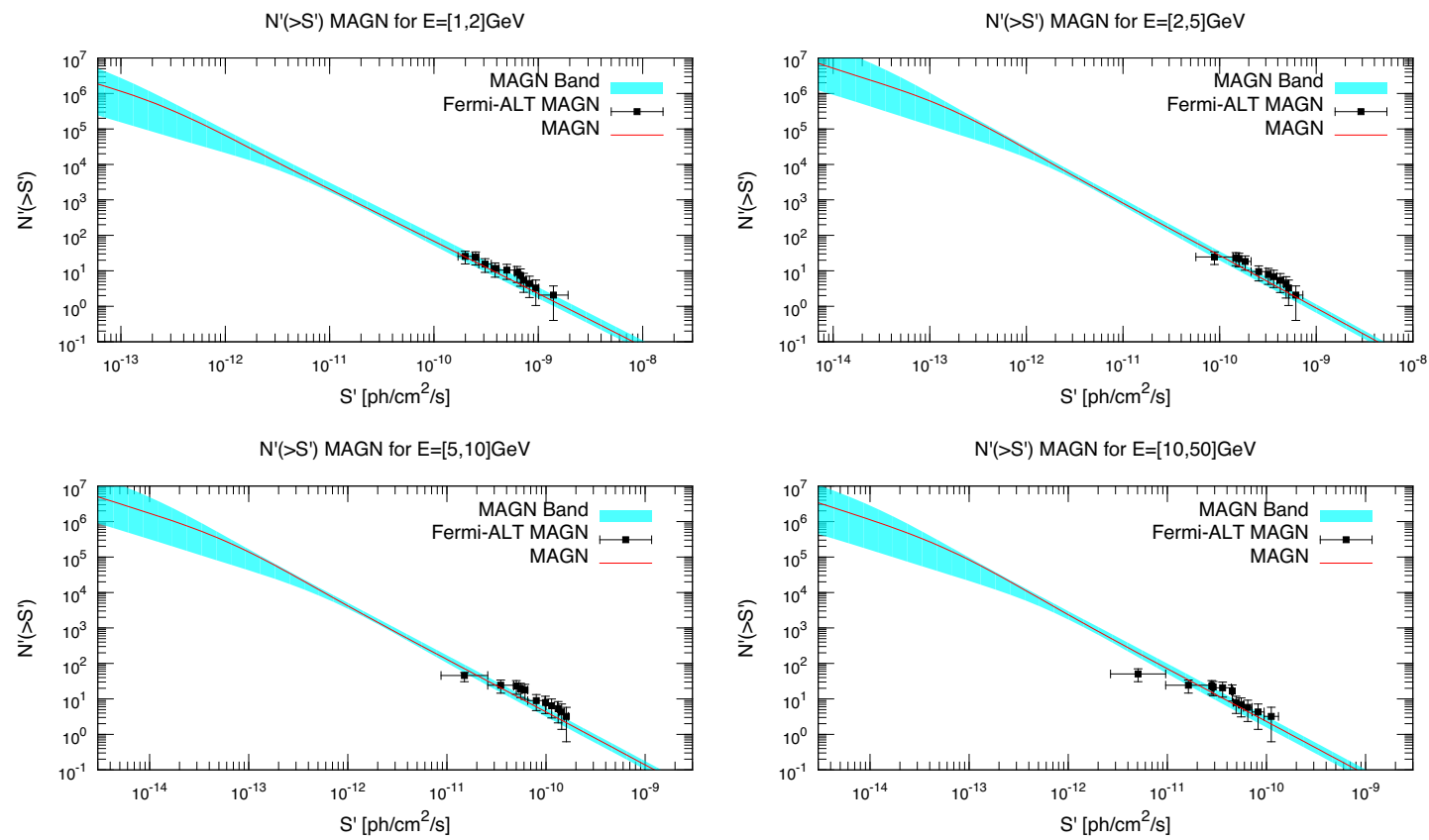

Figure 3. As in Fig. 2, but for MAGN. 


\section{Results}

The $C_{\mathrm{P}}(E)$ calculated using the models and the method described in $\S 2.2$ and $\S 2.4$ are shown in Fig. 4. We report results for the four energy bins used by the Fermi-LAT Collaboration in [2] (1.04-1.99 GeV, 1.99-5.00 GeV, 5.0-10.4 GeV and 10.4-50.0 GeV). Results are shown for all the radio-loud AGN populations: FSRQs, BL Lacs, and MAGN, as well as their sum. For completeness the results are shown in two different ways, i.e., the anisotropy integrated in each energy bin $\left(C_{\mathrm{P}}(E)\right)$, and the quantity $E^{4} C_{\mathrm{P}}(E) /(\Delta E)^{2}$ which resembles a differential anisotropy spectrum. The uncertainty for each theoretical bin has been derived from the uncertainty on the source count distribution given by the cyan band of the $N(>S)$ of Figs. 2 and 3. More precisely, the $1 \sigma$ band of the $N(>S)$ has first been transferred to the $d^{2} N /(d S d \Gamma)$ distribution and then propagated to the $C_{\mathrm{P}}(E)$ through Eq. 2.1. In all the bins the population that gives the largest anisotropy is the HSP BL Lacs. Indeed, considering Figs. 2 and 3, the HSP BL Lac population has about a factor of 3-5 times more sources in the bin 1.04-1.99 GeV with respect to MAGN at flux values just below the threshold of Fermi-LAT, which is the flux range where the unresolved sources contribute the most to the anisotropy. This factor of 3-5 between the number of HSP BL Lacs and MAGN translates into the same factor for the angular power in the bin 1.04-1.99 GeV (see Fig. 4).

We show in Fig. 5 the quantity $E^{4.5} C_{\mathrm{P}}(E) /(\Delta E)^{2}$ on a linear scale in order to illustrate more clearly the differences between the data and the theoretical predictions. We see that radio-loud AGN can account for the total anisotropy measured in [2] by the Fermi-LAT Collaboration, the data and model predictions being compatible within the errors. For example, in the energy range $1-10 \mathrm{GeV}$, the total theoretical expectation for the anisotropy from radio-loud AGN is $C_{\mathrm{P}}=9.3_{-2.5}^{+3.5} \cdot 10^{-18}\left(\mathrm{~cm}^{-2} \mathrm{~s}^{-1} \mathrm{sr}^{-1}\right)^{2}$ sr while the Fermi-LAT measurement is $(11.0 \pm 1.2) \cdot 10^{-18}\left(\mathrm{~cm}^{-2} \mathrm{~s}^{-1} \mathrm{sr}^{-1}\right)^{2} \mathrm{sr}$. However, a small trend (but within the errors) is visible, indicating that there could be room for further populations of sources contributing to the anisotropies below $2 \mathrm{GeV}$, while above $10 \mathrm{GeV}$ the anisotropy seems to be slightly overpredicted by the model. In this respect, a natural way to produce an anisotropy contribution only around a $\mathrm{GeV}$ would be to assume some contribution to the IGRB from unresolved millisecond pulsars [17]. Nevertheless, in Ref. [16] it has been recently shown that unresolved galactic millisecond pulsars contribute by less than the percent level to the measured anisotropy in each energy range. We note also that we neglect the possible contribution from cascading $\mathrm{GeV}$ emission from hard-spectrum sources induced by propagation of the $\mathrm{TeV}$ photons in the extra-galactic background light (EBL) $[54,55]$. This component is in principle sensitive to the presence of inter-galactic magnetic fields [55]. However, the presence itself of the cascade emission is still debated due to the possible damping effect of plasma instabilities (see [56-58]). Given the above uncertainties we will not investigate further this component.

We check the robustness of the results by comparing the expected anisotropy associated with different models. In particular the BL Lac models explored in [6], like our benchmark BL Lac model of [5], are not tuned explicitly to the anisotropy data and thus are suitable for a cross-check. The models explored in [6] use various different $\rho_{\gamma}$ parametrized as PLE 

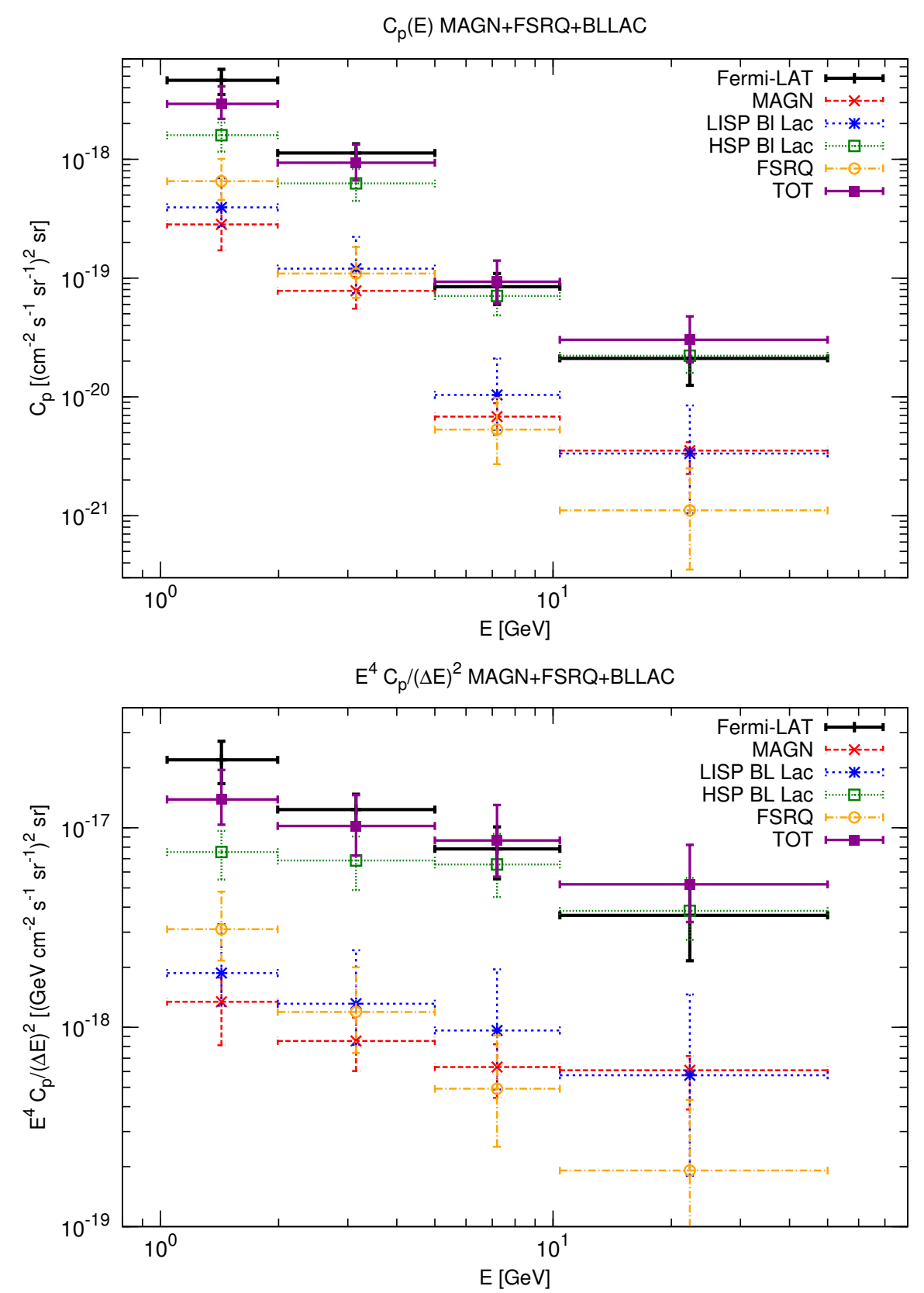

Figure 4. The angular power $C_{\mathrm{P}}(E)$ for MAGN (red long-dashed points), LISP (blue shortdashed) and HSP BL Lacs (green dotted), FSRQs (yellow dot-dashed), and the total anisotropy (violet solid) from all the radio-loud AGN is shown in two different units $\left(C_{\mathrm{P}}(E)\right.$ in the top panel and $E^{4} C_{\mathrm{P}}(E) /(\Delta E)^{2}$ in the bottom panel). The data measured in the four energy bins analyzed by the Fermi-LAT Collaboration [2] are also shown (black solid points).

(pure luminosity evolution), PDE (pure density evolution) or LDDE (luminosity dependent density evolution). We adopt the $\mathrm{PLE}_{3}$ model from Tab. 2 of [6].

The model derived in [37] adopts an LDDE $\gamma$-ray luminosity function based on the LF 


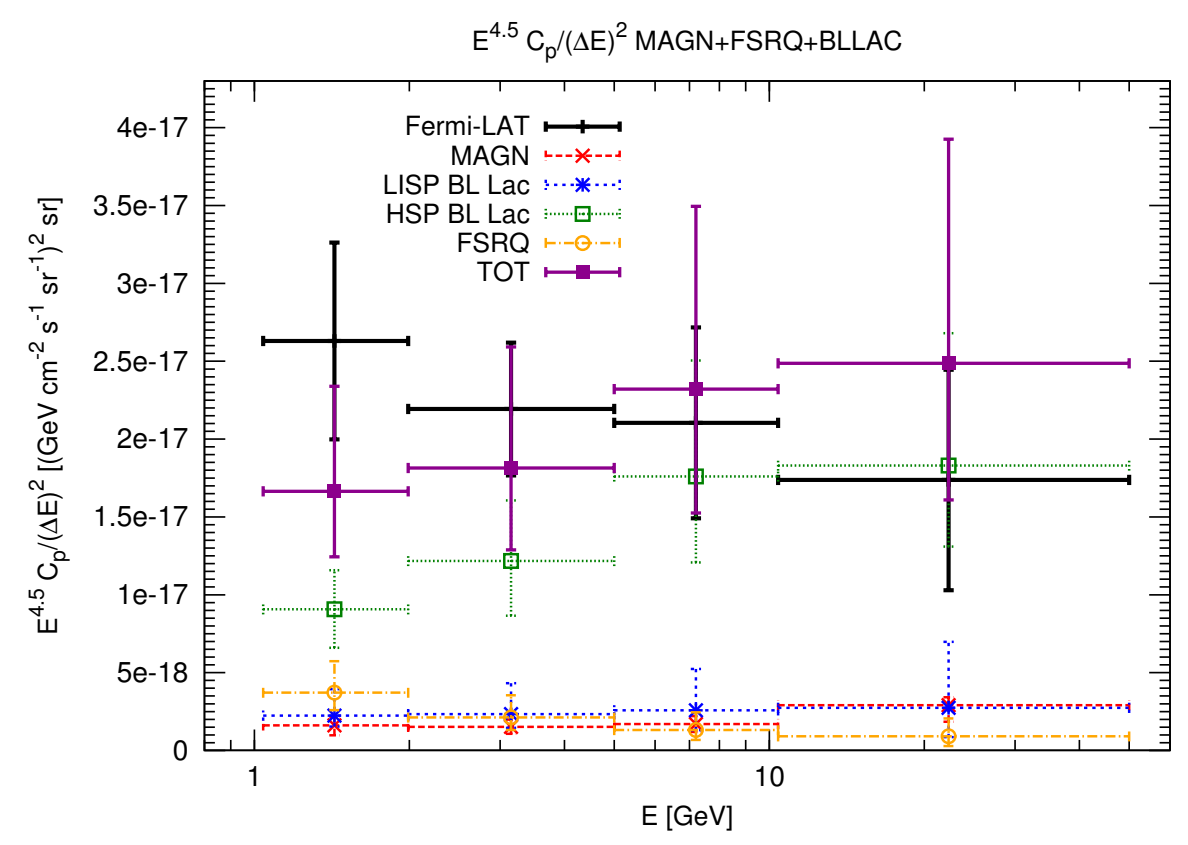

Figure 5. The angular power $C_{\mathrm{P}}(E)$, in units of $E^{4.5} C_{\mathrm{P}}(E) /(\Delta E)^{2}$, for MAGN (red long-dashed points), LISP (blue short-dashed) and HSP BL Lacs (green dotted), FSRQs (yellow dot-dashed), and the total anisotropy (violet solid) from all the radio-loud AGN is shown. The data measured in the four energy bins analyzed by the Fermi-LAT Collaboration [2] are also shown (black solid points).

of observed X-ray AGNs, which is then rescaled to $\gamma$ rays through a $L_{\gamma}-L_{X}$ relation. The SED, instead, is based on a SED blazar sequence model tuned to EGRET data. The free parameters of the model are then tuned to the observed anisotropy [2], resulting in a good match to the data themselves. The predicted IGRB intensity above $1 \mathrm{GeV}$ by this model is about $5 \%$ which is similar (a bit lower) to the predictions of [5] and [6] of about $15-20 \%$.

On the other hand $[38,56]$ studied a new $\gamma$-ray propagation model associated with plasma instabilities of ultra-relativistic $e^{+} e^{-}$pairs. The instability then dissipates the kinetic energy of the $\mathrm{TeV} e^{+} e^{-}$pairs produced during the propagation of $\mathrm{TeV} \gamma$ rays, suppressing the development of the associated electro-magnetic cascade and heating the intergalactic medium. The effectiveness of the plasma instability mechanism is, nonetheless, still being investigated (see, e.g., [57, 58]). The blazar LF used in [56] is based on the LF of optical and X-ray observed quasars, rescaled to $\gamma$-ray energies while the blazar SED is modeled as a broken power-law. No parameter of the model is tuned to $\gamma$-ray data. This $a$ priori model matches well the intensity and spectrum of the IGRB above few GeVs, thus being compatible with $100 \%$ of the IGRB contrary to the models described above which associate to blazars only a small fraction of the IGRB. The model, indeed, largely overpredicts the anisotropy. The authors claim, however, that the IGRB anisotropy measured in [2] has been substantially under-estimated [59].

Finally, the model developed in [60] is difficult to use for comparison in the present 


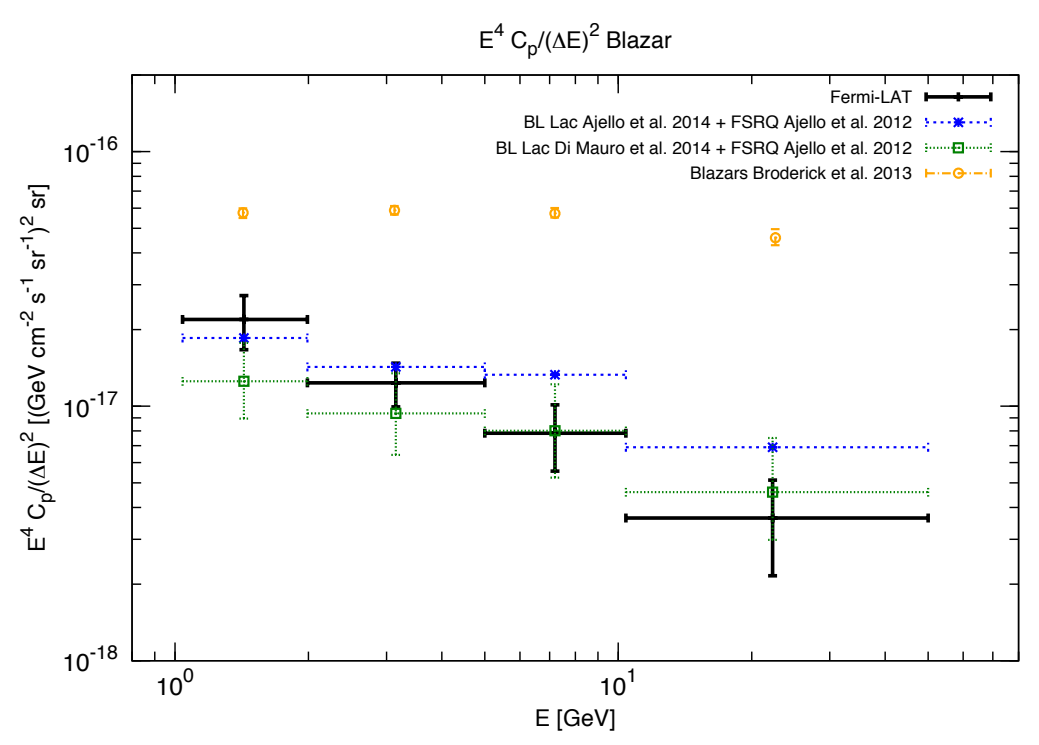

Figure 6. The angular power $C_{\mathrm{P}}(E)$ for different models of blazars is shown. Blue short-dashed: FSRQs from [4] and BL Lacs from [6]. Green dotted: BL Lacs from [5] and FSRQs from [4]. Orange circles: blazar anisotropies from [56]. The data measured in [1] by the Fermi-LAT Collaboration (black solid points) are also shown.

study since the full LF is not available. Nonetheless, the model has already been shown to be significantly in tension with the anisotropy data [35].

We show in Fig. 6 the anisotropy results for our benchmark case (FSRQs from [4] and BL Lacs from [5]) compared with the case of FSRQs from [4] and BL Lacs from [6], and the blazar model of [56], and with the observed anisotropy. We do not show the model anisotropies from [37] since after the fit they closely match the data. The model of [6] yields a larger anisotropy than the model of [5] in all energy bands, although still compatible with the measured Fermi-LAT anisotropy. Note that for the model of [6] we report the predicted anisotropy without uncertainties, adopting the central value for each of the parameters given in Tab. 2 of [6] for the $\mathrm{PLE}_{3}$ model without considering their errors. The parameters are in fact strongly correlated and propagating their uncertainty to the $C_{\mathrm{P}}$ would require knowledge of the full covariance matrix, which is not available. The differences between the $C_{\mathrm{P}}(E)$ in Fig. 6 are due to the differences in the $\gamma$-ray emission models and the catalogs used to calculate the average source parameters. In particular, the models for FSRQs and BL Lacs in [4, 6] use the 1FGL catalog [31] and use both an LDDE and PLE $\gamma$-ray luminosity function. The FSRQ SED is calibrated by combining Fermi-LAT data with X-ray measurements from the Swift Burst Alert Telescope (BAT) [61] while the BL Lac $\gamma$-ray SED is modeled using a simple power-law. The BL Lac model derived in [5] is calibrated using the 2FGL Fermi-LAT catalog [32] and uses both an LDDE and PLE $\gamma$-ray luminosity function. The $\gamma$-ray SED is studied by adding to the 2FGL catalog also the first Fermi-LAT catalog of Sources Above $10 \mathrm{GeV}$ (1FHL) [33] and the TeV measurements from available IACTs [62]. 


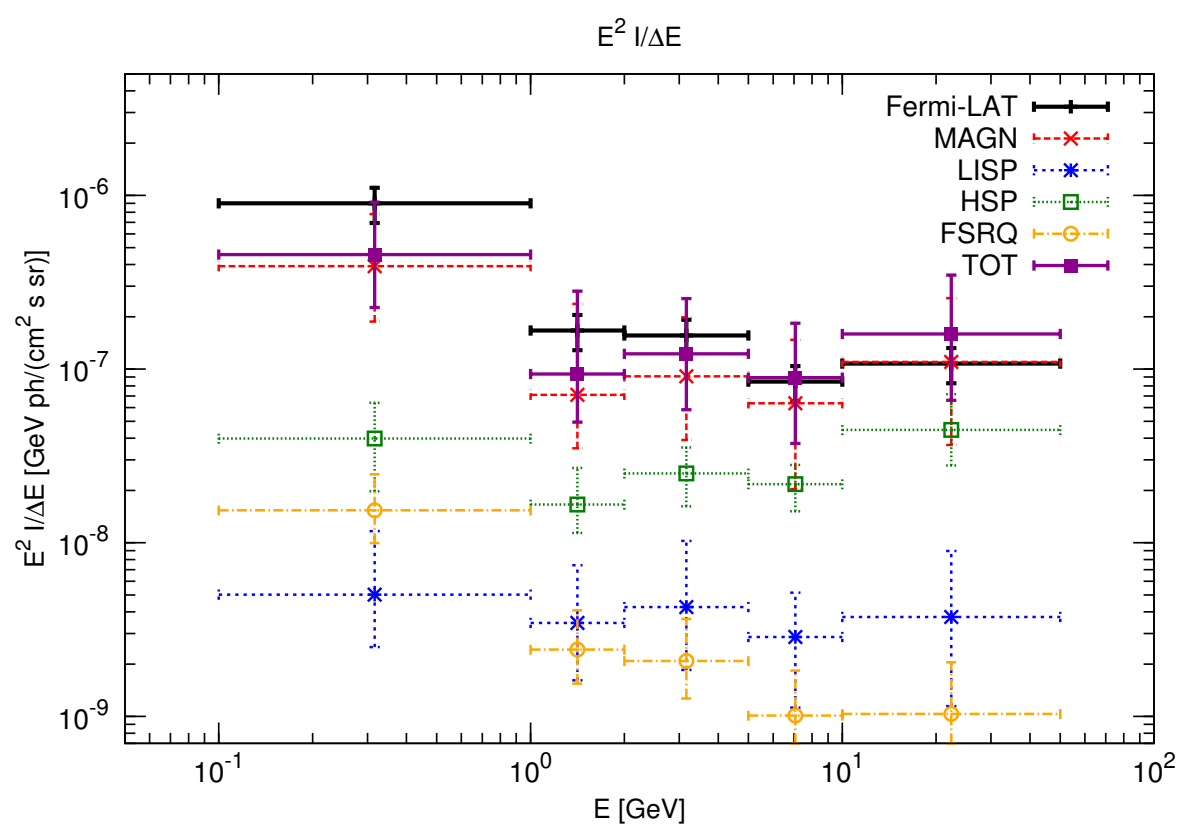

Figure 7. The integrated flux $E^{2} I / \Delta E$ for MAGN (red long-dashed points), LISP (blue shortdashed) and HSP BL Lacs (green dotted), FSRQs (yellow dot-dashed) and the total flux (violet solid) from all the radio-loud AGN are shown. Here we also show the data measured in [1] by the Fermi-LAT Collaboration rebinned into the 5 shown energy bins (black solid points).

Using the same formalism we can also calculate the expected flux contribution from radio-loud AGN to the IGRB [1]. The integrated $\gamma$-ray flux $I$ in a given energy bin can be written as:

$$
I\left(E_{0} \leq E \leq E_{1}\right)=\int_{\Gamma_{\min }}^{\Gamma_{\max }} d \Gamma \int_{0}^{S_{t}(\Gamma)} S \frac{d^{2} N}{d S d \Gamma} d S .
$$

The contribution to the IGRB intensity from MAGN, FSRQs and BL Lacs are shown in Fig. 7 with the same notation and in the same energy bins as Fig. 4. For a visual comparison with the anisotropy results we rebin the Fermi-LAT data of [1] into 5 new energy bins, 4 of which match the $C_{P}$ binning. The rebinned data are used only for visual purposes in Fig. 7. We emphasize that the radio-loud AGN emission can explain both the angular power and the intensity flux measured by Fermi-LAT, given that these two observables trace different features of the $\gamma$-ray population. Indeed the population that gives the largest contribution to anisotropy is, as we have already stressed, HSP BL Lacs because the unresolved part near the flux threshold of the Fermi-LAT is more populated. On the other hand, concerning the IGRB intensity, MAGN sources give the largest $\gamma$-ray flux, as already found in [5], because at fluxes much below the Fermi-LAT sensitivity, the MAGN population is expected to have many more sources with respect to the other radioloud AGN populations. Moreover FSRQ and LISP BL Lac sources provide only a small fraction of both the anisotropy and flux of the IGRB at energies larger than a few tens of $\mathrm{GeV}$ since their emission is suppressed by the cut-off or by the steep spectrum of their 


\begin{tabular}{||c|c|c|c|c|c|c|c||}
\hline Energy $(\mathrm{GeV})$ & LISP BL Lac & HSP BL Lac & BL Lac & FSRQ & Blazars & MAGN & AGN \\
\hline \hline $1.04-1.99$ & $8.5_{-2.7}^{+6.5}$ & $34.5_{-9.4}^{+9.5}$ & $43_{-12}^{+16}$ & $14.1_{-4.3}^{+7.7}$ & $57_{-16}^{+24}$ & $6.1_{-2.4}^{+2.0}$ & $63_{-19}^{+26}$ \\
$1.99-5.00$ & $10.6_{-4.0}^{+9.1}$ & $55.5_{-16.7}^{+17.7}$ & $66_{-20}^{+27}$ & $9.7_{-3.7}^{+6.5}$ & $76_{-24}^{+33}$ & $6.9_{-2.2}^{+2.2}$ & $83_{-26}^{+35}$ \\
$5.0-10.4$ & $12.3_{-6.6}^{+12 .}$ & $83.7_{-26.2}^{+35.4}$ & $96_{-32}^{+48}$ & $6.3_{-3.1}^{+5.4}$ & $102_{-35}^{+53}$ & $8.1_{-2.4}^{+2.4}$ & $110_{-38}^{+56}$ \\
$10.4-50.0$ & $15.7_{-10.8}^{+24.3}$ & $105_{-30}^{+49}$ & $121_{-41}^{+73}$ & $5.3_{-3.5}^{+6.6}$ & $126_{-44}^{+80}$ & $16.7_{-6.1}^{+3.0}$ & $143_{-50}^{+82}$ \\
\hline \hline
\end{tabular}

Table 1. Model predictions, together with $1 \sigma$ uncertainties, for the contribution to the IGRB anisotropy from the unresolved LISP BL Lacs, HSP BL Lacs, total from BL Lacs, FSRQs, all blazars $($ BL Lacs + FSRQs), MAGN, and all radio-loud AGN (blazars + MAGN) in the four energy bins indicated in the first column. The predictions are given in terms of the percentage of the central value of the experimental measure.

SED.

Anisotropy and flux are two complementary observables that can be used together to set constraints on the radio-loud AGN $\gamma$-ray emission. In Tab. 1 and 2 we display the best-fit and $\pm 1 \sigma$ percentage values of the contribution of each unresolved population to the measured IGRB anisotropy and intensity $[1,2]$. These percentage values are derived with respect to the central values of the data. As already pointed out above, radio-loud AGN sources can in principle entirely explain both the anisotropy and intensity data within the theoretical and experimental $1 \sigma$ uncertainties. However the uncertainty associated with the diffuse $\gamma$-ray emission from MAGN is large, due to the small sample of MAGN present in the Fermi-LAT 2FGL catalog [32]. The $\gamma$-ray flux from these objects could be more severely constrained by future Fermi-LAT catalogs with a larger number of sources and with a deeper knowledge of the correlation between the $\gamma$-ray and radio emission from the center of those sources.

LISP BL Lacs and FSRQs have a subdominant contribution to both the IGRB intensity and anisotropy, while MAGN give the largest contribution to the IGRB intensity and HSP BL Lacs the largest contribution to the anisotropy. The radio-loud AGN population gives the smallest fractional contribution to $C_{\mathrm{P}}(E)$ and the measured intensity in the lowest energy bins, namely at $1-2 \mathrm{GeV}$ for the anisotropy and $0.1-1 \mathrm{GeV}$ for the intensity, where the central values of the theoretical predictions are approximately half of the central value of the data. At these energies other populations could give a sizable contribution to the anisotropy and the diffuse emission. Star-forming galaxies have been widely studied and are expected to contribute in the range $0.1-10 \mathrm{GeV}$, see [11] and references therein. Millisecond pulsars, the most numerous Galactic population, have been shown to give negligible contribution to both the IGRB intensity and anisotropy [16].

Finally, one has to note that with increasing statistics a new measurement of the anisotropy will likely be performed in smaller energy bins and in an extended energy range. For reference, we thus provide predictions in the range $500 \mathrm{MeV}-200 \mathrm{GeV}$ in 12 energy bins. Results are shown in Fig. 8, where the energy dependence of the different populations is more clearly apparent. Again, as for Fig. 4, we show predictions in two complementary ways. The top panel shows the total anisotropy integrated in each energy bin $\left(C_{\mathrm{P}}(E)\right)$, 


\begin{tabular}{||c|c|c|c|c|c|c|c||}
\hline Energy $(\mathrm{GeV})$ & LISP BL Lac & HSP BL Lac & BL Lac & FSRQ & Blazars & MAGN & AGN \\
\hline \hline $0.1-1$ & $0.56_{-0.28}^{+0.73}$ & $4.4_{-2.2}^{+2.7}$ & $5.0_{-2.5}^{+3.4}$ & $1.7_{-0.6}^{+1.0}$ & $6.7_{-3.1}^{+4.5}$ & $43_{-23}^{+43}$ & $50_{-26}^{+48}$ \\
$1.04-1.99$ & $2.1_{-1.1}^{+2.4}$ & $10.0_{-3.1}^{+6.2}$ & $12_{-4}^{+9}$ & $1.5_{-0.5}^{+1.0}$ & $14_{-5}^{+10}$ & $43_{-22}^{+100}$ & $56_{-26}^{+109}$ \\
$1.99-5.00$ & $2.7_{-1.5}^{+3.8}$ & $16.0_{-5.7}^{+6.6}$ & $19_{-7}^{+10}$ & $1.3_{-0.5}^{+1.0}$ & $20_{-8}^{+11}$ & $58_{-33}^{+69}$ & $78_{-41}^{+80}$ \\
$5.0-10.4$ & $3.4_{-2.1}^{+2.7}$ & $26_{-7}^{+8}$ & $29_{-10}^{+10}$ & $1.2_{-0.5}^{+1.0}$ & $30_{-10}^{+11}$ & $75_{-51}^{+100}$ & $105_{-61}^{+110}$ \\
$10.4-50.0$ & $3.5_{-2.4}^{+1.9}$ & $41_{-16}^{+25}$ & $45_{-18}^{+30}$ & $0.96_{-0.53}^{+0.94}$ & $46_{-19}^{+31}$ & $102_{-68}^{+136}$ & $148_{-87}^{+167}$ \\
\hline \hline
\end{tabular}

Table 2. As in Table 1, but for the IGRB intensity.

while the bottom panel shows $E^{4} C_{\mathrm{P}}(E) /(\Delta E)^{2}$. It can be seen that at high energies, above about $10 \mathrm{GeV}$, only MAGN and HSP BL Lac sources still give a sizable contribution, due to the FSRQ and LISP BL Lac SED cut-off above these energies. The dip-like feature at about $1 \mathrm{GeV}$ in the top panel is due to the fine subdivision of the $1-2 \mathrm{GeV}$ range into 4 bins, consequently partitioning the total anisotropy in 4 bins. The effect disappears in the bottom panel differential spectrum where each bin is weighted by its energy width.

\section{Predicted anisotropy for CTA}

In this section we derive a forecast for the anisotropy from unresolved sources expected in CTA observations. To derive these predictions we use the same models and formalism considered in the previous sections extrapolated in the TeV energy range. CTA $[49,50]$ is a project in the development phase which will study very high-energy $\gamma$-rays from a few tens of $\mathrm{GeV}$ up to possibly $100 \mathrm{TeV}$. We use as benchmarks four different values of the CTA flux threshold (see Eq. 2.1) for point source detection, in units of the Crab flux, namely 5, 10, 50 and 100 mCrab. To define the Crab flux we assume a power-law spectra with a photon index of 2.63 normalized to a flux of $3.45 \cdot 10^{-11} \mathrm{~cm}^{-2} \mathrm{~s}^{-1} \mathrm{TeV}^{-1}$ at $1 \mathrm{TeV}$ as measured by [63]. We consider predictions in the energy range $[0.1,10] \mathrm{TeV}$, divided into four energy bins (0.1-0.3, 0.3-1, 1-3 and 3-10 TeV) in which we have computed the unresolved angular power for radio-loud AGN varying the flux threshold in the range $[5,100]$ mCrab flux. For simplicity, we do not consider any index bias, but we assume a single threshold in each energy bin independent from the spectral index $\Gamma$. We expect the anisotropy variation due to different thresholds to be much larger than spectral index bias, which, in any case, is difficult to model at present without precise knowledge of CTA's instrumental response functions.

The anisotropy predictions are shown in Fig. 9 for the separated MAGN, LISP and HSP BL Lac, and FSRQ populations while their sum is shown in Fig. 10. As already noticed for Fig. 8, above $100 \mathrm{GeV}$ the anisotropy is dominated by the HSP and MAGN populations while LISP and FSRQs provide a sub-dominant contribution. The CTA experiment could perform observations both in single-source pointing mode and in survey mode. With the single-source configuration the flux sensitivity is a few $\mathrm{mCrab}$, while in the survey strategy it could be a few tens of mCrab [50]. Hence, in the first case the unresolved angular power for $E>100 \mathrm{GeV}$ could be of the order of $10^{-22}\left(\mathrm{~cm}^{-2} \mathrm{~s}^{-1} \mathrm{sr}^{-1}\right)^{2} \mathrm{sr}$. On the other hand, 
$\mathrm{C}_{\mathrm{p}}(\mathrm{E}) \mathrm{MAGN}+\mathrm{FSRQ}+\mathrm{BLLAC}$

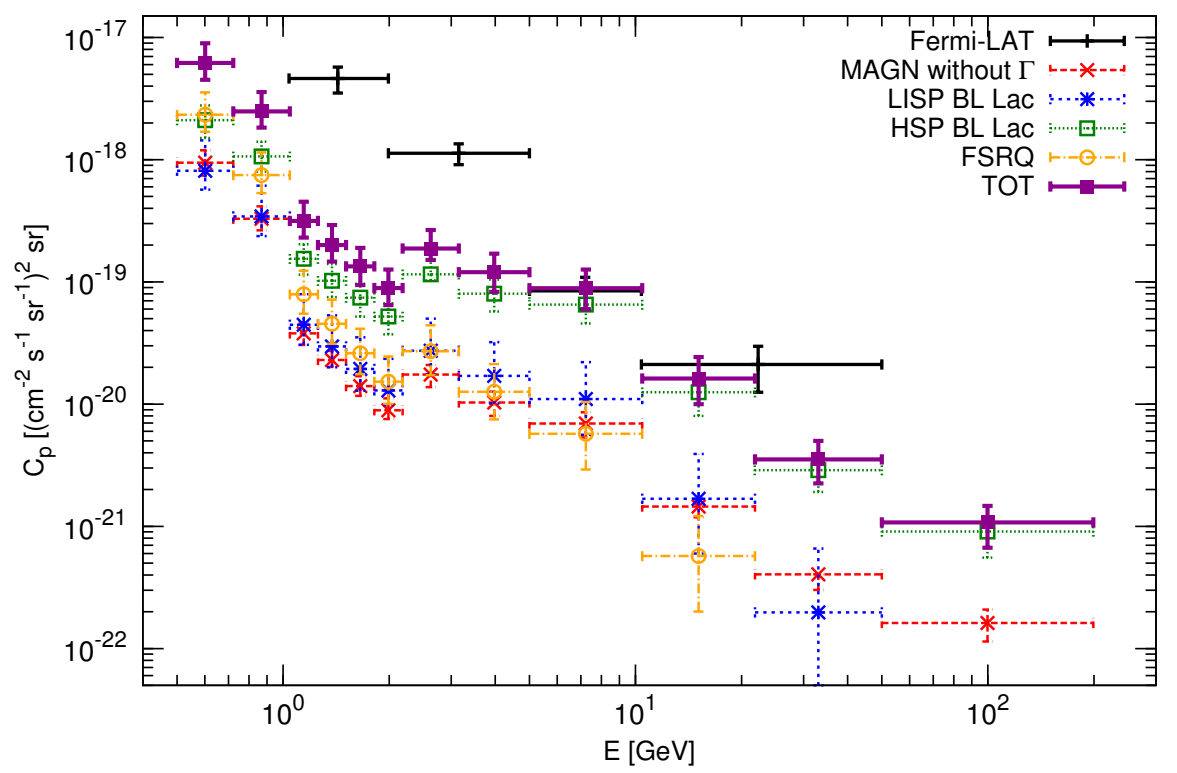

$E^{4} C_{p} /(\Delta E)^{2} M A G N+F S R Q+B L L A C$

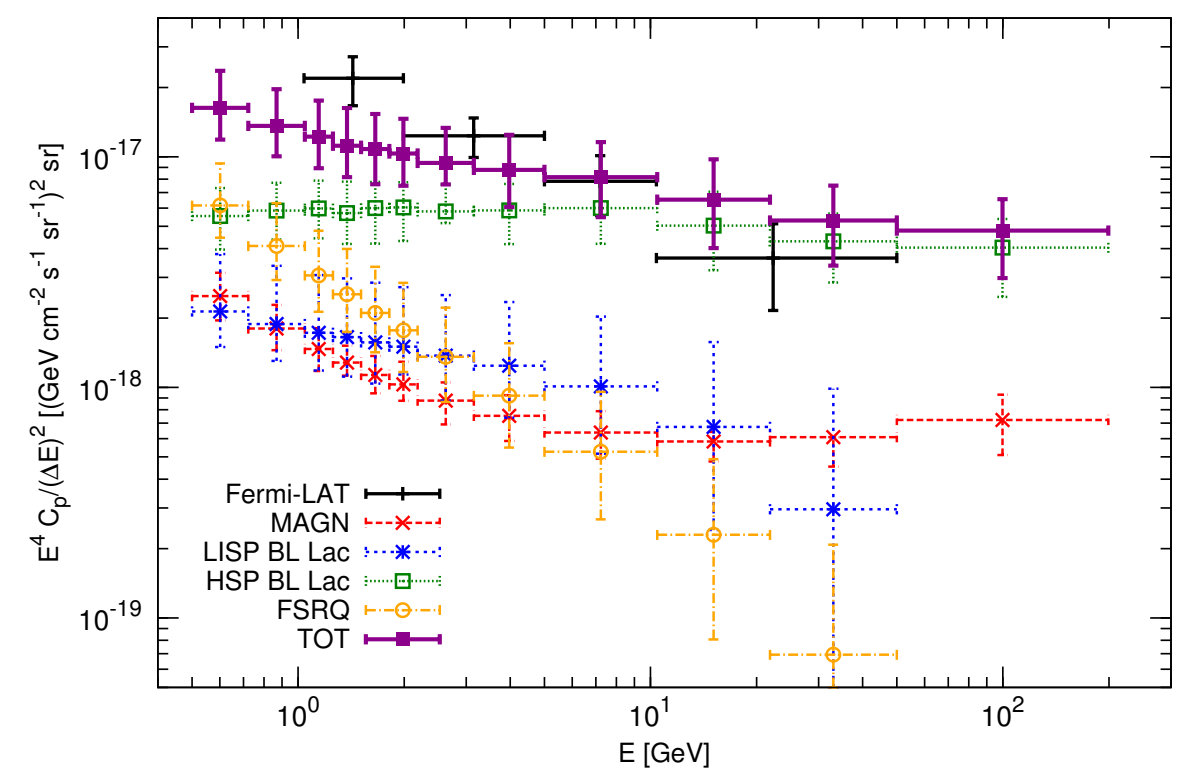

Figure 8. In this figure we display the angular power $C_{\mathrm{P}}(E)$, in two different units $\left(C_{\mathrm{P}}(E)\right.$, top, and $E^{4} C_{\mathrm{P}}(E) /(\Delta E)^{2}$, bottom), for MAGN (red long-dashed), LISP (blue short- dashed) and HSP BL Lac (green dotted), FSRQ (yellow dot-dashed) and the total anisotropy (violet solid) in 12 energy bins, from 0.5 to $200 \mathrm{GeV}$. For illustrative purposes we also show the Fermi- LAT data [2] (grey solid points). Note that in the top panel the quantity shown is integrated within each energy bin, and thus its value depends on the size of the bin considered; the binning differs between the measured data points and the predictions.

with survey mode observations the unresolved angular power for $E>100 \mathrm{GeV}$ could be in the range $\left[10^{-22}, 10^{-21}\right]\left(\mathrm{cm}^{-2} \mathrm{~s}^{-1} \mathrm{sr}^{-1}\right)^{2} \mathrm{sr}$. 

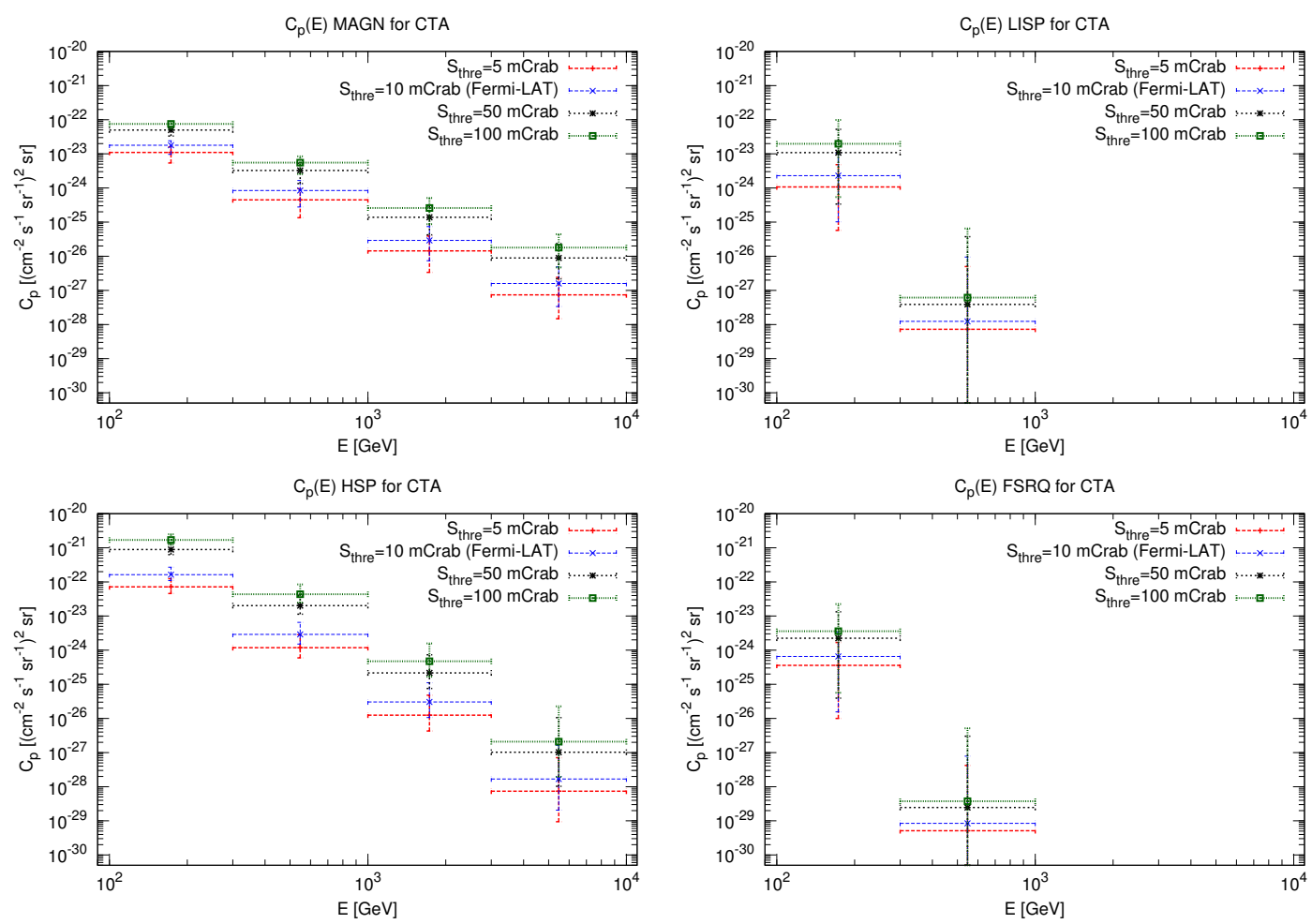

Figure 9. Predicted angular power $C_{\mathrm{P}}(E)$ for different expected CTA sensitivities (in units of the Crab flux), in 4 energy bins from $100 \mathrm{GeV}$ to $10 \mathrm{TeV}$. The top left and top right panels refer to unresolved MAGN and LISPs, respectively, while the bottom left and bottom right panels refer to HSP and FSRQ sources, respectively.

A way to make these predictions more intuitive is to compare CTA's sensitivity with that of the Fermi-LAT, at least in the energy range $10-1000 \mathrm{GeV}$ where there is a partial overlap between the two instruments' operating energies. Looking at the sensitivity map in the First High Energy Fermi- Catalog (1FHL) [33], the flux sensitivity at high latitudes $(b>10)$ is about $S_{f}=9 \cdot 10^{-11} \mathrm{ph} / \mathrm{cm}^{2} / \mathrm{s}$ for energies larger than $10 \mathrm{GeV}$. On the other hand, the Crab flux above $10 \mathrm{GeV}$ for the power-law model assumed above is $8.4 \cdot 10^{-9}$ $\mathrm{ph} / \mathrm{cm}^{2} / \mathrm{s}$ which translates to a Fermi-LAT sensitivity of about $10 \mathrm{mCrab}$. For comparison, we thus indicate in Figs. 9 and 10 the 10 mCrab sensitivity as "Fermi-LAT-equivalent sensitivity".

Finally, we show in Fig. 11 also the fluctuation angular power as function of energy, i.e. the quantity $C_{\mathrm{P}} /\langle I\rangle^{2}$, where $\langle I\rangle$ is the mean intensity of the emission, which is another useful measure of the anisotropy properties of the unresolved emission $[2,46]$. In particular, for a given threshold flux and a given energy bin, $C_{\mathrm{P}}$ and $I$ are given by Eqs. 2.1 and 3.1, which means that $C_{\mathrm{P}} /\langle I\rangle^{2}$ could have a non-trivial behavior as function of the threshold flux since both $C_{\mathrm{P}}$ and $I$ vary with it. It can be seen that below $100 \mathrm{GeV}$ the $C_{\mathrm{P}} /\langle I\rangle^{2}$ curve is approximately flat around $\sim 10^{-4}$. This value is in agreement with the value of $\sim 10^{-5}$ measured with the Fermi-LAT in [2], after accounting for the fact that the $\langle I\rangle$ used 


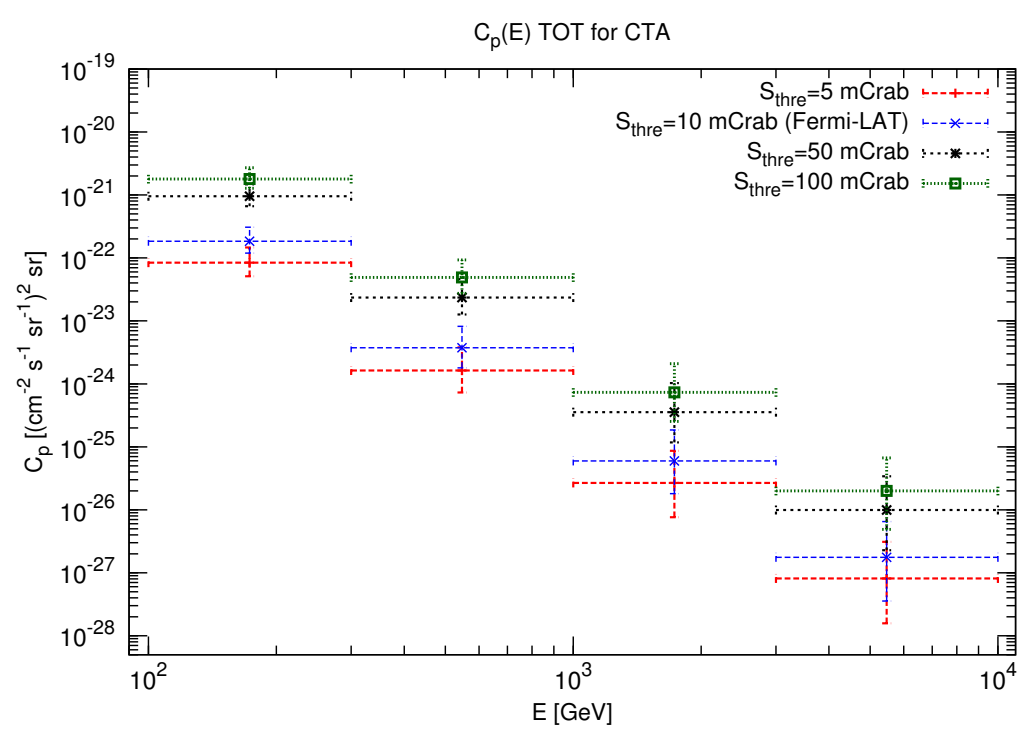

Figure 10. Total angular power $C_{\mathrm{P}}(E)$ from all the unresolved radio-loud AGN (sum of the contributions shown in Fig. 9) in the 4 energy bins listed in the text and for different threshold fluxes in units of the Crab flux.

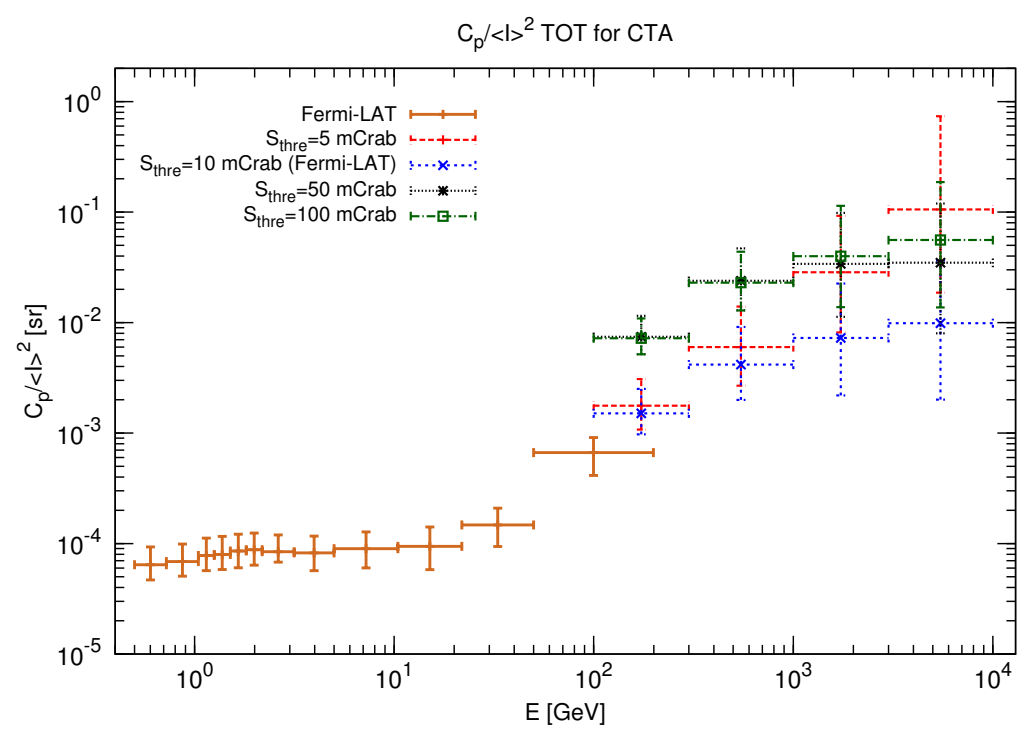

Figure 11. Total angular power (sum from all radio-loud AGN populations) divided by the square of the intensity $C_{\mathrm{P}} /\langle I\rangle^{2}$ for 4 high-energy bins and for different flux thresholds in units of the Crab flux, and for 12 additional energy bins in the range $500 \mathrm{MeV}-200 \mathrm{GeV}$ as in Fig. 8.

in [2] to calculate $C_{\mathrm{P}} /\langle I\rangle^{2}$ is the average high-latitude diffuse emission rather than the true IGRB, and the former is roughly a factor of 3 larger than the true IGRB due to the Galactic diffuse emission contribution. Above $100 \mathrm{GeV} C_{\mathrm{P}} /\langle I\rangle^{2}$ has a clear upturn. This is due to the partial attenuation of the extragalactic $\gamma$-ray photons by the extra-galactic background light (EBL) due to $e^{+} e^{-}$pair production. This effect is taken into account in 

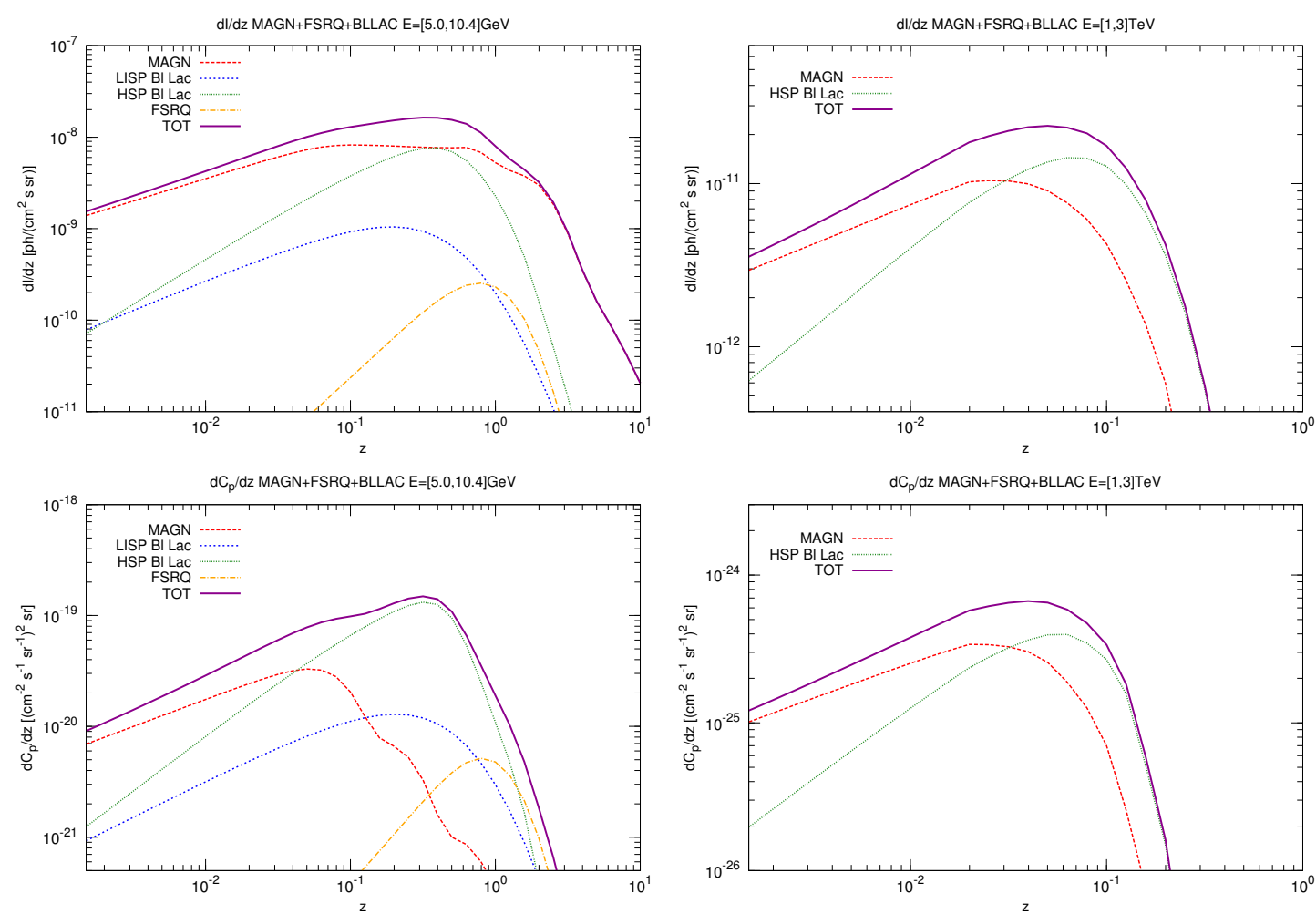

Figure 12. IGRB intensity distribution $d I / d z$ and anisotropy distribution $d C_{P} / d z$ as function of redshift $z$. The curves show the separate contributions from each component and the total. The left column shows the intensity and anisotropy in the 5-10.4 GeV energy bin, while the right column refers to the 1-3 TeV energy bin and for a detection threshold of $10 \mathrm{mCrab}$.

our calculations using the EBL model of [64]. As cross-check we also verified that our results do not change significantly when using the alternative EBL attenuation models of [65-67]. All the above models are, indeed, in rough agreement among each other and with the observed high-energy spectra of blazars as seen by the Fermi-LAT and HESS [68, 69]. In practice, the EBL attenuation causes a steepening of the IGRB spectrum by absorbing the high-redshift, high-energy contribution to the IGRB. The high-redshift part of the IGRB is also typically the most isotropic since, due to volume effects, is made by many distant (and thus faint) sources, so that it gives only a minor contribution to $C_{\mathrm{P}}$. For this reason, the anisotropy $C_{\mathrm{P}}$ is not significantly affected by the EBL, in contrast to the intensity $\langle I\rangle$ which is more strongly affected, so that the ratio $C_{\mathrm{P}} /\langle I\rangle^{2}$ increases with energy. This effect is illustrated in Fig. 12, where the IGRB intensity distribution $d I / d z$ and anisotropy distribution $d C_{P} / d z$ are shown as function of redshift $z$ for the two energy bins 5-10.4 GeV and 1-3 TeV. At energies of few GeVs, it can be seen indeed that while the IGRB intensity is produced at redshifts up to 3-4, the anisotropy originates basically from more nearby redshifts of $z \lesssim 1$. At TeV energies, instead, where the effect of the EBL absorption is significant, both the intensity and anisotropy originates from nearby redshifts $z \lesssim 0.1$. 
Using $C_{\mathrm{P}} /\langle I\rangle^{2}$ we can also compare our results to the sensitivity study of IACTs to anisotropies. In [70] anisotropies from both dark matter and astrophysical sources have been studied in the two energy ranges $E>100 \mathrm{GeV}$ and $E>300 \mathrm{GeV}$. It is shown that CTA should be sensitive to anisotropies in the astrophysical component down to a value as low as $10^{-5}$. A value of $\sim 10^{-3}$ as predicted by our analysis above $100 \mathrm{GeV}$ should thus be easily detectable with CTA. Indeed, a value as high as $\sim 10^{-3}$ could already be within the reach of present day IACTs. On the other hand, a value of $\sim 10^{-3}$ could present a challenge for dark matter searches in the $\mathrm{TeV}$ range since in [70] an astrophysical background of $\sim 10^{-5}$ was assumed. A re-analysis of the sensitivity of IACTs to dark-matter-induced anisotropy considering the present up-to-date models of the radio-loud AGN population will be required to accurately understand the potential of these instruments to use anisotropies to search for dark matter.

\subsection{Discussion}

As already noted above, the anisotropy at CTA energies, above $100 \mathrm{GeV}$, is dominated by HSP BL Lacs and MAGNs. The predicted intensity and anisotropy depends on the SED of these populations which, at these high energies, is still affected by various uncertainties due to the scarcity of sources observed. For example, the effect of the assumed energy cutoff of HSPs of $910 \mathrm{GeV}$ is clearly visible in Fig. 9, with the anisotropy in the last bin being considerably suppressed. On the other hand, considering the 1- $\sigma$ uncertainties in [5], the energy cutoff can be in principle in the range $460-2010 \mathrm{GeV}$. Variation of the cutoff within this range will correspondingly affect the level of anisotropy, especially in the 2 highest-energy bins. For MAGNs a cutoff is not observed, while, instead, at least in the case of Cen A, a hardening above $\sim 10 \mathrm{GeV}$ is seen [71]. If this is a general feature of MAGNs, a corresponding enhancement in the anisotropy from MAGNs at high energies should be expected. Again, due to very limited number of sources detected at $\mathrm{TeV}$ energies, a robust conclusion is not possible. Finally, some further classes of $\mathrm{TeV}$ sources might be present. For example, extreme HBLs, sometimes dubbed ultra-high-frequency-peaked BL Lac $[72,73]$, are BL Lacs with the synchrotron peak at MeV energies and they show at IACTs energies a hardening of the spectra. As discussed in [5], 20\% of the HSP sample does not show a cut off in the spectra and this sample could be part of this extreme class of BL Lacs. Although the sample is not homogenous, it can be reasonably assumed that ultra-high-frequency-peaked BL Lacs represent around $20 \%$ of HSP population. This class of BL Lacs could thus contribute to enhance the anisotropy at $\mathrm{TeV}$ energies. Clearly, to fully address this issue, larger statistics of BL Lacs sources at $\mathrm{TeV}$ energies are required. The situation should become more clear in the following years, as more sources will be hopefully detected.

Finally, we add some consideration on the effective detectability of the IGRB intensity and anisotropy with CTA. This issue is discussed, to some extent, in [70]. Due to the large amount of CR background, especially electrons, in IACT observations, the signal-to-noise ratio for the IGRB intensity measurement is quite low and the prospects for a measurement are limited. On the contrary, somewhat counter-intuitively, detection of IGRB anisotropies might be feasible since the CR background is not expected to exhibit small scale anisotropies 
giving a larger signal-to-noise. In [70], the prospects for IGRB anisotropy detection with CTA have been investigated in a simplified setup and found to be promising (see also discussion in the previous section). A deeper study using detailed simulations of CTA observations will help to spot potential unseen problematics and eventually to confirm the possibility of anisotropy observations with CTA.

\section{Conclusions}

In this work we have studied the contribution of various classes of radio-loud AGN to the intensity and anisotropy of the IGRB. We have used up-to-date phenomenological models for the $\gamma$-ray luminosity functions of these populations to predict the $\gamma$-ray flux and anisotropy as functions of the energy. We have found that the entirety of the IGRB intensity and anisotropy as measured by the Fermi-LAT can be explained by radio-loud AGN, with MAGN providing the bulk of the measured intensity but a low anisotropy, while high-synchrotron-peaked BL Lac objects give the main contribution to the anisotropy. Nonetheless, the predicted intensity from MAGN still suffers from large uncertainties, which are difficult to reduce even with the use of anisotropy information, given the low level of anisotropy predicted from this population.

Within these uncertainties, there may be still some room for further $\gamma$-ray populations, provided that their anisotropy remains low in order not to exceed the measurements, which are already fairly saturated by the HSP BL Lac contribution. In this respect, for example, star-forming galaxies could still provide a substantial contribution to the low-energy side of the IGRB, while exhibiting a low level of anisotropy and keeping a self-consistent picture in agreement with the observed IGRB intensity and anisotropy.

Upcoming analyses of 5 years of Fermi-LAT data below about $100 \mathrm{GeV}$ and forthcoming observations with the Cherenkov Telescope Array above $100 \mathrm{GeV}$ will provide valuable tests of the AGN scenario depicted in the present analysis, and will contribute significantly to improving our knowledge of high-energy $\gamma$-ray emitters.

\section{Acknowledgments}

We thank Pasquale D. Serpico and Hannes Zechlin for useful discussions and a careful reading of the manuscript. This work is supported by the research grant TAsP (Theoretical Astroparticle Physics) funded by the Istituto Nazionale di Fisica Nucleare (INFN), by the Strategic Research Grant: Origin and Detection of Galactic and Extragalactic Cosmic Rays funded by Torino University and Compagnia di San Paolo, by the Spanish MINECO under grants FPA2011-22975 and MULTIDARK CSD2009-00064 (Consolider-Ingenio 2010 Programme), by Prometeo/2009/091 (Generalitat Valenciana), and by the EU ITN UNILHC PITN-GA-2009-237920. At LAPTh this activity was supported by the Labex grant ENIGMASS. JSG acknowledges support from NASA through Einstein Postdoctoral Fellowship grant PF1-120089 awarded by the Chandra X-ray Center, which is operated by the Smithsonian Astrophysical Observatory for NASA under contract NAS8-03060. 


\section{References}

[1] Fermi-LAT Collaboration, A. Abdo et al., The Spectrum of the Isotropic Diffuse Gamma-Ray Emission Derived From First-Year Fermi Large Area Telescope Data, Phys.Rev.Lett. 104 (2010) 101101, [arXiv:1002.3603].

[2] Fermi-LAT Collaboration, M. Ackermann et al., Anisotropies in the diffuse gamma-ray background measured by the Fermi LAT, Phys.Rev. D85 (2012) 083007, [arXiv:1202.2856].

[3] M. Di Mauro, F. Calore, F. Donato, M. Ajello, and L. Latronico, Diffuse $\gamma$-ray emission from misaligned active galactic nuclei, Astrophys.J. 780 (2014) 161, [arXiv:1304.0908].

[4] M. Ajello, M. Shaw, R. Romani, C. Dermer, L. Costamante, et al., The Luminosity Function of Fermi-detected Flat-Spectrum Radio Quasars, Astrophys.J. 751 (2012) 108, [arXiv:1110.3787].

[5] M. Di Mauro, F. Donato, G. Lamanna, D. Sanchez, and P. Serpico, Diffuse $\gamma$-ray emission from unresolved BL Lac objects, Astrophys.J. 786 (2014) 129, [arXiv:1311.5708].

[6] M. Ajello, R. Romani, D. Gasparrini, M. Shaw, J. Bolmer, et al., The Cosmic Evolution of Fermi BL Lacertae Objects, Astrophys.J. 780 (2014) 73, [arXiv:1310.0006].

[7] Y. Inoue, Contribution of Gamma-Ray-loud Radio Galaxies' Core Emissions to the Cosmic MeV and GeV Gamma-Ray Background Radiation, ApJ 733 (May, 2011) 66, [arXiv: 1103.3946].

[8] F. W. Stecker and M. H. Salamon, The Gamma-Ray Background from Blazars: A New Look, ApJ 464 (June, 1996) 600, [astro-ph/9601120].

[9] C. D. Dermer, Statistics of Cosmological Black Hole Jet Sources: Blazar Predictions for the Gamma-Ray Large Area Space Telescope, ApJ 659 (Apr., 2007) 958-975, [astro-ph/0605402].

[10] Y. Inoue and T. Totani, The Blazar Sequence and the Cosmic Gamma-ray Background Radiation in the Fermi Era, ApJ 702 (Sept., 2009) 523-536, [arXiv:0810.3580].

[11] Fermi-LAT Collaboration, M. Ackermann et al., GeV Observations of Star-forming Galaxies with Fermi LAT, Astrophys.J. 755 (2012) 164, [arXiv:1206.1346].

[12] B. D. Fields, V. Pavlidou, and T. Prodanović, Cosmic Gamma-ray Background from Star-forming Galaxies, ApJL 722 (Oct., 2010) L199-L203, [arXiv:1003.3647].

[13] I. Tamborra, S. Ando, and K. Murase, Star-forming galaxies as the origin of diffuse high-energy backgrounds: Gamma-ray and neutrino connections, and implications for starburst history, arXiv: 1404.1189.

[14] B. C. Lacki, S. Horiuchi, and J. F. Beacom, The Star-forming Galaxy Contribution to the Cosmic MeV and GeV Gamma-Ray Background, ApJ 786 (May, 2014) 40, [arXiv: 1206.0772].

[15] T. A. Thompson, E. Quataert, and E. Waxman, The Starburst Contribution to the Extra-Galactic Gamma-Ray Background, Astrophys.J. 654 (2006) 219-225, [astro-ph/0606665].

[16] F. Calore, M. Di Mauro, F. Donato, and F. Donato, Diffuse gamma-ray emission from galactic pulsars, Astrophys.J. 796 (2014) 1, [arXiv:1406.2706]. 
[17] J. M. Siegal-Gaskins, R. Reesman, V. Pavlidou, S. Profumo, and T. P. Walker, Anisotropies in the gamma-ray sky from millisecond pulsars, Mon.Not.Roy.Astron.Soc. 415 (2011) 1074S, [arXiv: 1011.5501].

[18] T. Grégoire and J. Knödlseder, Constraining the Galactic millisecond pulsar population using Fermi Large Area Telescope, A $B A 554$ (June, 2013) A62, [arXiv:1305.1584].

[19] T. Bringmann, F. Calore, M. Di Mauro, and F. Donato, Constraining dark matter annihilation with the isotropic $\gamma$-ray background: updated limits and future potential, Phys.Rev. D89 (2014) 023012, [arXiv: 1303.3284].

[20] M. Cirelli, P. Panci, and P. D. Serpico, Diffuse gamma ray constraints on annihilating or decaying Dark Matter after Fermi, Nucl.Phys. B840 (2010) 284-303, [arXiv:0912.0663].

[21] K. N. Abazajian, S. Blanchet, and J. P. Harding, Current and Future Constraints on Dark Matter from Prompt and Inverse-Compton Photon Emission in the Isotropic Diffuse Gamma-ray Background, Phys.Rev. D85 (2012) 043509, [arXiv:1011.5090].

[22] J. Zavala, V. Springel, and M. Boylan-Kolchin, Extragalactic gamma-ray background radiation from dark matter annihilation, Mon.Not.Roy.Astron.Soc. 405 (2010) 593, [arXiv: 0908.2428].

[23] I. Cholis, D. Hooper, and S. D. McDermott, Dissecting the Gamma-Ray Background in Search of Dark Matter, JCAP 1402 (2014) 014, [arXiv: 1312.0608].

[24] K. I. Kellermann, R. Sramek, M. Schmidt, D. B. Shaffer, and R. Green, VLA observations of objects in the Palomar Bright Quasar Survey, AJ 98 (Oct., 1989) 1195-1207.

[25] C. M. Urry and P. Padovani, Unified Schemes for Radio-Loud Active Galactic Nuclei, PASP 107 (Sept., 1995) 803, [astro-ph/].

[26] J. R. P. Angel and H. S. Stockman, Optical and infrared polarization of active extragalactic objects, ARA\&A 18 (1980) 321-361.

[27] P. Padovani and P. Giommi, The connection between x-ray- and radio-selected BL Lacertae objects, ApJ 444 (May, 1995) 567-581, [astro-ph/].

[28] Fermi-LAT Collaboration, A. A. Abdo et al., The Spectral Energy Distribution of Fermi Bright Blazars, ApJ 716 (June, 2010) 30-70, [arXiv:0912.2040].

[29] Fermi-LAT Collaboration, M. Ackermann et al., The Second Catalog of Active Galactic Nuclei Detected by the Fermi Large Area Telescope, ApJ 743 (2011) 171, [arXiv:1108.1420].

[30] G. Fossati, L. Maraschi, A. Celotti, A. Comastri, and G. Ghisellini, A unifying view of the spectral energy distributions of blazars, MNRAS 299 (Sept., 1998) 433-448, [astro-ph/9804103].

[31] Fermi-LAT Collaboration, A. Abdo et al., Fermi Large Area Telescope First Source Catalog, Astrophys.J.Suppl. 188 (2010) 405-436, [arXiv: 1002.2280].

[32] Fermi-LAT Collaboration, A. Abdo et al., Fermi Large Area Telescope Second Source Catalog, Astrophys.J.Suppl. 199 (2012) 31, [arXiv:1108.1435].

[33] Fermi-LAT Collaboration, A. Abdo et al., The First Fermi-LAT Catalog of Sources Above $10 \mathrm{GeV}$, arXiv:1306.6772.

[34] K. Bechtol, The Isotropic diffuse gamma-ray emission between $100 \mathrm{MeV}$ and $820 \mathrm{GeV}$, High Energy Messengers Workshop, Chicago, June 2014 (2014) [https://kicp-workshops.uchicago.edu/hem2014/depot/talk-bechtol-keith.pdf]. 
[35] A. Cuoco, E. Komatsu, and J. Siegal-Gaskins, Joint anisotropy and source count constraints on the contribution of blazars to the diffuse gamma-ray background, Phys.Rev. D86 (2012) 063004, [arXiv: 1202.5309].

[36] Fermi-LAT Collaboration, A. Abdo et al., The Fermi-LAT high-latitude Survey: Source Count Distributions and the Origin of the Extragalactic Diffuse Background, Astrophys.J. 720 (2010) 435-453, [arXiv: 1003.0895].

[37] J. P. Harding and K. N. Abazajian, Models of the Contribution of Blazars to the Anisotropy of the Extragalactic Diffuse Gamma-ray Background, JCAP 1211 (2012) 026, [arXiv: 1206.4734].

[38] C. Pfrommer, A. E. Broderick, P. Chang, E. Puchwein, and V. Springel, The physics and cosmology of TeV blazars in a nutshell, ArXiv e-prints (Aug., 2013) [arXiv:1308.6284].

[39] S. Ando and V. Pavlidou, Imprint of Galaxy Clustering in the Cosmic Gamma-Ray Background, Mon.Not.Roy.Astron.Soc. 400 (2009) 2122, [arXiv:0908.3890].

[40] S. Ando and E. Komatsu, Anisotropy of the cosmic gamma-ray background from dark matter annihilation, Phy. Rev. D 73 (Jan., 2006) 023521, [astro-ph/0512217].

[41] S. Ando, E. Komatsu, T. Narumoto, and T. Totani, Dark matter annihilation or unresolved astrophysical sources? Anisotropy probe of the origin of the cosmic gamma-ray background, Phy. Rev. D 75 (Mar., 2007) 063519, [astro-ph/0612467].

[42] J. M. Siegal-Gaskins, Revealing dark matter substructure with anisotropies in the diffuse gamma-ray background, JCAP 0810 (2008) 040, [arXiv:0807.1328].

[43] B. S. Hensley, J. M. Siegal-Gaskins, and V. Pavlidou, The detectability of dark matter annihilation with Fermi using the anisotropy energy spectrum of the gamma-ray background, Astrophys.J. 723 (2010) 277-284, [arXiv:0912.1854].

[44] S. Ando, Gamma-ray background anisotropy from Galactic dark matter substructure, Phy. Rev. D 80 (July, 2009) 023520, [arXiv:0903.4685].

[45] M. Fornasa, L. Pieri, G. Bertone, and E. Branchini, Anisotropy probe of galactic and extra-galactic dark matter annihilations, Phy. Rev. D 80 (July, 2009) 023518, [arXiv: 0901.2921].

[46] J. M. Siegal-Gaskins and V. Pavlidou, Robust Identification of Isotropic Diffuse Gamma Rays from Galactic Dark Matter, Physical Review Letters 102 (June, 2009) 241301, [arXiv: 0901.3776].

[47] A. Cuoco, A. Sellerholm, J. Conrad, and S. Hannestad, Anisotropies in the diffuse gamma-ray background from dark matter with Fermi LAT: a closer look, MNRAS $\mathbf{4 1 4}$ (July, 2011) 2040-2054, [arXiv: 1005.0843].

[48] F. Calore, V. De Romeri, M. Di Mauro, F. Donato, J. Herpich, et al., Gamma-ray anisotropies from dark matter in the Milky Way: the role of the radial distribution, Mon.Not.Roy.Astron.Soc. 442 (2014) 1151-1156, [arXiv:1402.0512].

[49] CTA Consortium Collaboration, M. Actis et al., Design concepts for the Cherenkov Telescope Array CTA: An advanced facility for ground-based high-energy gamma-ray astronomy, Exper.Astron. 32 (2011) 193-316, [arXiv:1008.3703].

[50] G. Dubus, J. Contreras, S. Funk, Y. Gallant, T. Hassan, et al., Surveys with the Cherenkov Telescope Array, Astropart.Phys. 43 (2013) 317-330, [arXiv:1208.5686]. 
[51] D. Scott and M. J. White, Implications of SCUBA observations for the Planck Surveyor, Astron.Astrophys. 346 (1999) 1, [astro-ph/9808003].

[52] D. W. Hogg, Distance measures in cosmology, astro-ph/9905116.

[53] C. J. Willott, S. Rawlings, K. M. Blundell, M. Lacy, and S. A. Eales, The radio luminosity function from the low-frequency 3CRR, 6CE and 7CRS complete samples, MNRAS $\mathbf{3 2 2}$ (Apr., 2001) 536-552, [astro-ph/].

[54] F. Aharonian, P. Coppi, and H. Volk, Very high-energy gamma-rays from AGN: Cascading on the cosmic background radiation fields and the formation of pair halos, Astrophys.J. 423 (1994) L5-L8, [astro-ph/9312045].

[55] T. M. Venters and V. Pavlidou, Probing the intergalactic magnetic field with the anisotropy of the extragalactic gamma-ray background, MNRAS 432 (July, 2013) 3485-3494, [arXiv: 1201.4405].

[56] A. E. Broderick, C. Pfrommer, E. Puchwein, and P. Chang, Implications of Plasma Beam Instabilities for the Statistics of the Fermi Hard Gamma-ray Blazars and the Origin of the Extragalactic Gamma-Ray Background, ArXiv e-prints (Aug., 2013) [arXiv:1308.0340].

[57] A. Saveliev, C. Evoli, and G. Sigl, The Role of Plasma Instabilities in the Propagation of Gamma-Rays from Distant Blazars, arXiv:1311.6752.

[58] L. Sironi and D. Giannios, Relativistic Pair Beams from TeV Blazars: A Source of Reprocessed GeV Emission rather than Intergalactic Heating, Astrophys.J. 787 (2014) 49, [arXiv: 1312.4538].

[59] A. E. Broderick, C. Pfrommer, E. Puchwein, and P. Chang, Lower Limits upon the Anisotropy of the Extragalactic Gamma-Ray Background implied by the 2FGL and 1FHL Catalogs, ArXiv e-prints (July, 2013) [arXiv: 1308.0015].

[60] F. W. Stecker and T. M. Venters, Components of the Extragalactic Gamma-ray Background, ApJ 736 (July, 2011) 40, [arXiv: 1012.3678].

[61] N. Gehrels et al., The Swift Gamma-Ray Burst Mission, ApJ 611 (Aug., 2004) 1005-1020.

[62] S. Wakely and H. Deirdre http: // tevcat. uchicago. edu (2013).

[63] H.E.S.S. Collaboration Collaboration, F. Aharonian et al., Observations of the Crab Nebula with H.E.S.S, Astron.Astrophys. 457 (2006) 899-915, [astro-ph/0607333].

[64] J. D. Finke, S. Razzaque, and C. D. Dermer, Modeling the Extragalactic Background Light from Stars and Dust, ApJ 712 (Mar., 2010) 238-249, [arXiv:0905.1115].

[65] A. Franceschini, G. Rodighiero, and M. Vaccari, The extragalactic optical-infrared background radiations, their time evolution and the cosmic photon-photon opacity, Astron.Astrophys. 487 (2008) 837, [arXiv:0805.1841].

[66] R. Gilmore, R. Somerville, J. Primack, and A. Dominguez, Semi-analytic modeling of the EBL and consequences for extragalactic gamma-ray spectra, arXiv:1104.0671.

[67] A. Dominguez, J. Primack, D. Rosario, F. Prada, R. Gilmore, et al., Extragalactic Background Light Inferred from AEGIS Galaxy SED-type Fractions, arXiv:1007.1459.

[68] Fermi-LAT Collaboration, M. Ackermann et al., The Imprint of The Extragalactic Background Light in the Gamma-Ray Spectra of Blazars, Science 338 (2012) 1190-1192, [arXiv:1211.1671]. 
[69] HESS Collaboration, A. Abramowski et al., Measurement of the extragalactic background light imprint on the spectra of the brightest blazars observed with H.E.S.S, A\&A 550 (2012) [arXiv:1212.3409].

[70] J. Ripken, A. Cuoco, H.-S. Zechlin, J. Conrad, and D. Horns, The sensitivity of Cherenkov telescopes to dark matter and astrophysically induced anisotropies in the diffuse gamma-ray background, JCAP 1401 (2014) 049, [arXiv: 1211.6922].

[71] N. Sahakyan, R. Yang, F. Aharonian, and F. Rieger, Evidence for a second component in the high-energy core emission from Centaurus A?, Astrophys.J. 770 (2013) L6, [arXiv: 1302.7173].

[72] G. Sentrk, M. Errando, M. Bttcher, and R. Mukherjee, Gamma-Ray Observational Properties of TeV-Detected Blazars, Astrophys.J. 764 (2013) 119, [arXiv:1301.3697].

[73] E. Lefa, F. Rieger, and F. Aharonian, Formation of hard very-high energy spectra of blazars in leptonic models, Astrophys.J. $\mathbf{7 4 0}$ (2011) 64, [arXiv:1106.4201]. 